\title{
ON THE BRAUER-PICARD GROUPS OF FUSION CATEGORIES
}

\author{
IAN MARSHALL AND DMITRI NIKSHYCH
}

\begin{abstract}
We develop methods of computation of the Brauer-Picard groups of fusion categories and apply them to compute such groups for several classes of fusion categories of prime power dimension: representation categories of elementary abelian groups with twisted associativity, extra special $p$-groups, and the Kac-Paljutkin Hopf algebra. We conclude that many finite groups of Lie type occur as composition factors of the Brauer-Picard groups of pointed fusion categories.
\end{abstract}

\section{INTRODUCTION}

Let $\mathcal{A}$ be a fusion category. The $\operatorname{Brauer-Picard} \operatorname{group} \operatorname{BrPic}(\mathcal{A})$ was introduced in ENO1 as the group of equivalence classes of invertible $\mathcal{A}$-bimodule categories. The motivation for this definition is that given a finite group $G$ and a $G$-graded fusion category $\mathcal{B}=\oplus_{g \in G} \mathcal{B}_{g}$ whose trivial component $\mathcal{B}_{1}$ is equivalent to $\mathcal{A}$ there is a group homomorphism

$$
G \rightarrow \operatorname{BrPic}(\mathcal{A})
$$

and, conversely, any such $\mathcal{B}$ can be constructed from a homomorphism (1) and some cohomological data. It was also shown in ENO1 that there is a canonical isomorphism

$$
\operatorname{BrPic}(\mathcal{A}) \cong \operatorname{Aut}^{b r}(\mathcal{Z}(\mathcal{A})),
$$

where $\operatorname{Aut}^{b r}(\mathcal{Z}(\mathcal{A}))$ is the group of isomorphism classes of braided tensor autoequivalences of the center $\mathcal{Z}(\mathcal{A})$ of $\mathcal{A}$. The latter group can be thought of as a categorical analogue of an orthogonal group. For example, if $\mathcal{A}$ is the representation category of a finite abelian group $A$, one has $\operatorname{Aut}^{b r}(\mathcal{Z}(\mathcal{A})) \cong O(A \oplus \widehat{A})$, where $\widehat{A}$ is the group of characters of $A$ and $O(A \oplus \widehat{A})$ is the group of automorphisms of $A \oplus \widehat{A}$ preserving its canonical quadratic form.

The definition of $\operatorname{BrPic}(\mathcal{A})$ is rather abstract, while the group $\operatorname{Aut}^{b r}(\mathcal{Z}(\mathcal{A}))$ is defined in more concrete terms and, hence, is easier to compute. There is also an important geometric intuition coming from the action of $\operatorname{Aut}^{b r}(\mathcal{Z}(\mathcal{A}))$ on Lagrangian subcategories of the center (these are categorical counterparts of Lagrangian subspaces). Such actions were used in $\overline{B N}, \overline{N R}, \mathrm{R}$ to compute Brauer-Picard groups of various symmetric tensor categories. An analogue of the Bruhat decomposition of $\operatorname{BrPic}(\operatorname{Rep}(G))$, where $G$ is a finite group, was recently discussed in $[\mathrm{LP}$.

Let $p$ be a prime. By a fusion p-category we mean a fusion category whose Frobenius-Perron dimension is a power of $p$. For odd $p$ such a category is always integral, i.e., is equivalent to the representation category of some semisimple quasiHopf algebra GN], while for $p=2$ it is a $\mathbb{Z}_{2}$-extension of an integral fusion category.

Fusion $p$-categories were classified in [DGNO1] (see also [EGNO, Section 9.14]). Namely, it was shown that any integral fusion $p$-category $\mathcal{A}$ is group-theoretical, 
i.e., it is categorically Morita equivalent to a pointed fusion category. Explicitly, this means that $\mathcal{A}$ is equivalent to the category of bimodules over an algebra in the category $\mathcal{C}(G, \omega)$ of vector spaces graded by a $p$-group $G$ with the associativity constraint given by $\omega \in H^{3}\left(G, k^{\times}\right)$. Thus, all fusion $p$-categories can be described in terms of $p$-groups and their cohomology.

In this paper we develop methods of computing the group $\operatorname{BrPic}(\mathcal{A})$, where $\mathcal{A}$ is a fusion category, using the induction homomorphism

$$
\text { ind : } \operatorname{Aut}(\mathcal{A}) \rightarrow \operatorname{Aut}^{b r}(\mathcal{Z}(\mathcal{A})) \cong \operatorname{BrPic}(\mathcal{A})
$$

from the group of tensor autoequivalences of $\mathcal{A}$ to the group of braided autoequivalences of its center, see Section 3 for a precise definition. The image of this homomorphism is a subgroup of $\operatorname{BrPic}(\mathcal{A})$ which may or may not coincide with the whole group. If $\tilde{\mathcal{A}}$ is a fusion category categorically Morita equivalent to $\mathcal{A}$ (so that $\operatorname{BrPic}(\mathcal{A}) \cong \operatorname{Aut}^{b r}(\mathcal{Z}(\tilde{\mathcal{A}}))$ then one can also induce central autoequivalences of $\mathcal{Z}(\mathcal{A})$ from $\tilde{\mathcal{A}}$. Thus, in general, there are several induction homomorphisms that can be combined to compute the Brauer-Picard group. Another useful tool of constructing elements of the Brauer-Picard group is the Picard induction of an invertible $\mathcal{Z}(\mathcal{A})$-module category (i.e., a $\mathcal{A}$-bimodule category) from an invertible category over a subcategory $\mathcal{D} \subset \mathcal{Z}(\mathcal{A})$.

We use these methods to begin a systematic study of Brauer-Picard groups of fusion $p$-categories. These groups turn out to be rather large and interesting. In particular, they include extensions of many classical finite groups of Lie type. Since the Brauer-Picard group is invariant under categorical Morita equivalence, it suffices to compute the Brauer-Picar groups of categories $\mathcal{A}=\mathcal{C}(G, \omega)$, where $G$ is a $p$ group. We explicitly compute these groups in the cases when $G$ is an elementary abelian or extra special $p$-group. We also compute the Brauer-Picard group of the representation category of the celebrated Kac-Paljutkin Hopf algebra KP.

The paper is organized as follows.

In Section 2 we recall definitions and basic facts about Brauer-Picard groups and tensor autoequivalences of fusion categories.

In Section 3 we study the induction homomorphism (3). Proposition 3.1 gives an exact sequence that can be used to compute the kernel of ind. We characterize the image of induction in Theorem 3.3 as the stabilizer of a certain Lagrangian algebra in the center. The orbits of the action of the Brauer-Picard group on the categorical Lagrangian Grassmannian are described in Section 3.4 .

In Section 4 we prove several results about the structure of representation categories of twisted quantum doubles (i.e., categories $\mathcal{Z}(\mathcal{C}(G, \omega))$ ). In particular, we analyze the image of the above induction homomorphism in this case and give a sufficient conditions for it to be surjective (Corollary 4.7). We also note a useful braided equivalence (30) that allows to replace the group $G$ by an easier group.

In Section 5 for an elementary abelian $p$-group $V$ we describe the cohomology group $H^{3}\left(V, k^{\times}\right)$as a $G L(V)$-module and obtain exact sequences containing the Brauer-Picard groups of categories $\mathcal{C}(V, \omega)$. We show that under certain nondegeneracy conditions these Brauer-Picard groups are abelian extensions of stabilizers of certain elements in exterior and symmetric algebras of $V^{*}$.

Finally, in Section 6 we compute the Brauer-Picard groups of several classes of fusion $p$-categories: representation categories of extra special $p$-groups, pointed 
fusion categories coming from simple modular Lie algebras, and the representation category of the Kac-Paljutkin Hopf algebra of dimension 8.

Acknowledgments We are grateful to Derek Holt, Geoffrey Mason, Viktor Ostrik, and Leonid Vainerman for helpful discussions. We thank Cesar Galindo for useful discussions and for pointing the paper GP to us. We thank Ehud Meir for explaining to us the classification of module categories over TambaraYamagami categories from [MM] and sharing his calculations with us. The authors were partially supported by the NSA grant H98230-15-1-0003. The second author was partially supported by the NSA grant H98230-16-1-0008.

\section{Preliminaries}

2.1. Fusion categories and Brauer-Picard groups. In this paper we work over an algebraically closed field $k$ of characteristic 0 . By a fusion category we mean a semisimple rigid tensor category with finitely many isomorphism classes of simple objects and finite dimensional spaces of morphisms. For basic results of the theory of fusion categories see [EGNO, DGNO2].

For a fusion category $\mathcal{A}$ let $\operatorname{Aut}(\mathcal{A})$ denote the group of isomorphism classes of tensor autoequivalences of $\mathcal{A}$. If $\mathcal{A}$ is a braided fusion category we denote $\operatorname{Aut}^{b r}(\mathcal{A})$ the group of isomorphism classes of braided tensor autoequivalences of $\mathcal{A}$.

For a group $G$ let Aut $(G)$ denote the group of automorphisms of $G$, let $\operatorname{lnn}(G)$ denote the subgroup of inner automorphisms, and let $\operatorname{Out}(G)=\operatorname{Aut}(G) / \operatorname{Inn}(G)$ be the group of (classes of) outer automorphisms of $G$.

A fusion category is called pointed if all its simple objects are invertible with respect to the tensor product. For a fusion category $\mathcal{A}$ let $\mathcal{A}_{p t}$ denote its maximal pointed fusion subcategory, i.e., the fusion subcategory generated by invertible simple objects of $\mathcal{A}$.

Any pointed fusion category is equivalent to some category $\mathcal{C}(G, \omega)$ of vector spaces graded by a finite group $G$ with the associativity constraint given by a 3cocycle $\omega \in H^{3}\left(G, k^{\times}\right)$. The center $\mathcal{Z}(\mathcal{C}(G, \omega))$ is known to be equivalent to the representation category of the twisted group double, i.e., the quasi-Hopf algebra $D^{\omega}(G)$.

Remark 2.1. A common notation for $\mathcal{C}(G, \omega)$ in the literature is $\operatorname{Vec}_{G}^{\omega}$. We choose a different notation in order to keep subscripts reasonable.

The Brauer-Picard group $\operatorname{BrPic}(\mathcal{A})$ of a fusion category $\mathcal{A}$ consists of equivalence classes of invertible $\mathcal{A}$-bimodule categories [ENO1] with multiplication given by the tensor product over $\mathcal{A}$. It was shown in ENO1, ENO2 that there is a canonical isomorphism

$$
\Phi: \operatorname{BrPic}(\mathcal{A}) \stackrel{\sim}{\rightarrow} \operatorname{Aut}^{b r}(\mathcal{Z}(\mathcal{A})),
$$

where $\mathcal{Z}(\mathcal{A})$ denotes the center of $\mathcal{A}$.

More generally, for a braided fusion category $\mathcal{C}$ the Picard group of $\mathcal{C}$ consists of equivalence classes of one-sided $\mathcal{C}$-module categories. When $\mathcal{C}$ is non-degenerate, there is an isomorphism

$$
\Phi: \operatorname{Pic}(\mathcal{C}) \stackrel{\sim}{\rightarrow} \operatorname{Aut}^{b r}(\mathcal{C}) .
$$

Note that (4) is a special case of (5) $\operatorname{since} \operatorname{Pic}(\mathcal{Z}(\mathcal{A})) \cong \operatorname{BrPic}(\mathcal{A})$.

Isomorphism (4) was used in [NR] to analyze Brauer-Picard groups of categories $\mathcal{C}(G, 1)=\operatorname{Vec}_{G}$ and compute them in concrete examples. 
2.2. Fusion $p$-categories. Let $p$ be a prime integer. By a fusion p-category we mean a fusion category whose Frobenius-Perron dimension is $p^{n}$ for some integer $n$. Such categories were characterized in [DGNO1] (see also [EGNO, Section 9.4]). Namely, any such category $\mathcal{A}$ which is integral (i.e., such that every object of $\mathcal{A}$ has an integral Frobenius-Perron dimension) is group-theoretical, i.e., there is a $p$-group $G$ and $\omega \in H^{3}\left(G, k^{\times}\right)$such that $\mathcal{A}$ is categorically Morita equivalent to $\mathcal{C}(G, \omega)$. In particular,

$$
\operatorname{BrPic}(\mathcal{A}) \cong \operatorname{BrPic}(\mathcal{C}(G, \omega)) .
$$

Remark 2.2. When $p$ is odd, a fusion $p$-category is automatically integral.

Computations of Brauer-Picard groups of concrete "small" examples of fusion $p$-categories were given in $\mathrm{NR}$. In particular, it was shown that

$$
\operatorname{BrPic}(\mathcal{C}(D, 1))=S_{4}, \quad \operatorname{BrPic}(\mathcal{C}(Q, 1))=S_{3},
$$

where $D$ and $Q$ are, respectively, the dihedral and quaternion groups of order 8 and $S_{n}$ denotes the symmetric group of degree $n$.

Furthermore, it was shown in $[\mathrm{R}$, that if $G$ is the (unique) non-abelian group of order $p^{3}$ and exponent $p$, where $p$ is odd, then

$$
\operatorname{BrPic}(\mathcal{C}(G, 1))=S L_{3}\left(\mathbb{F}_{p}\right) \text {. }
$$

2.3. Tensor autoequivalences of pointed fusion categories. Let $G$ be a finite group and let $\omega \in H^{3}\left(G, k^{\times}\right)$be a 3 -cocycle.

Let $\operatorname{Stab}(\omega) \subset \operatorname{Aut}(G)$ be the subgroup of automorphisms $a \in \operatorname{Aut}(G)$ such that $\omega \circ(a \times a \times a)$ and $\omega$ are cohomologous. Note that $\operatorname{Inn}(G) \subset \operatorname{Stab}(\omega)$. The following result is well known (and is easy to check).

Proposition 2.3. There is a short exact sequence

$$
0 \rightarrow H^{2}\left(G, k^{\times}\right) \rightarrow \operatorname{Aut}(\mathcal{C}(G, \omega)) \rightarrow \operatorname{Stab}(\omega) \rightarrow 0 .
$$

Here $H^{2}\left(G, k^{\times}\right)$parameterizes the tensor functor structures on the identity endofunctor of $\mathcal{C}(G, \omega)$. Given $a \in \operatorname{Stab}(\omega)$ and a 2-cochain $\mu$ such that

$$
d^{2}(\mu)=\frac{\omega \circ(a \times a \times a)}{\omega}
$$

let $F_{a, \mu}$ denote the corresponding autoequivalence of $\mathcal{C}(G, \omega)$.

2.4. Lagrangian subcategories. Let $\mathcal{C}$ be a non-degenerate braided fusion category. A fusion subcategory $\mathcal{L} \subset \mathcal{C}$ is called Lagrangian if $\mathcal{L}$ is Tannakian, i.e., $\mathcal{L}=\operatorname{Rep}(G)$ for some finite group $G$, and $\mathcal{L}$ coincides with its Müger centralizer, i.e., $\mathcal{L}=\mathcal{L}^{\prime}$. In this case the regular algebra $A=\operatorname{Fun}(G)$ of $\operatorname{Rep}(G)$ is a Lagrangian algebra in $\mathcal{C}$ (see Section 3.3) and the category $\mathcal{C}_{A}$ of left $A$-modules in $\mathcal{C}$ is equivalent to $\mathcal{C}(G, \omega)$ for some $\omega \in H^{3}\left(G, k^{\times}\right)$, so that there is a braided tensor equivalence

$$
\mathcal{C} \cong \mathcal{Z}(\mathcal{C}(G, \omega))
$$

See [DGNO2, Section 4.4.10] for details. 
2.5. Properties of the Picard induction. Let $\mathcal{C}$ be a braided fusion category and let $\mathcal{D} \subset \mathcal{C}$ be a fusion subcategory. There is a homomorphism of Picard groups given by the induction:

$$
P_{\mathcal{D}}^{\mathcal{C}}: \operatorname{Pic}(\mathcal{D}) \rightarrow \operatorname{Pic}(\mathcal{C}): \mathcal{M} \mapsto \mathcal{C} \otimes_{\mathcal{D}} \mathcal{M}
$$

We will call this homomorphism the Picard induction. A different kind of induction (of central autoequivalences) will be considered in Section 3 .

The kernel of $P_{\mathcal{D}}^{\mathcal{C}}$ was described in [BN], Proposition 3.11]. Namely, let

$$
\mathcal{C}=\bigoplus_{\alpha \in \Sigma} \mathcal{C}_{\alpha}
$$

be the decomposition of $\mathcal{C}$ into a direct sum of $\mathcal{D}$-module subcategories. Then

$$
\operatorname{Ker}\left(P_{\mathcal{D}}^{\mathcal{C}}\right)=\left\{\mathcal{C}_{\alpha}, \alpha \in \Sigma \mid \mathcal{C}_{\alpha} \text { is an invertible } \mathcal{D} \text {-module category }\right\}
$$

\section{INDUCTION OF CENTRAL AUTOEQUIVALENCES}

3.1. Induction $\operatorname{Aut}(\mathcal{A}) \rightarrow \operatorname{Aut}^{b r}(\mathcal{Z}(\mathcal{A}))$. Let $\mathcal{A}$ be a fusion category. Recall that the objects of the center $\mathcal{Z}(\mathcal{A})$ of $\mathcal{A}$ are pairs $(Z, \gamma)$, where $Z$ is an object of $\mathcal{A}$ and $\gamma=\left\{\gamma_{X}: X \otimes Z \rightarrow Z \otimes X\right\}$ is a natural isomorphism satisfying certain coherence axioms, see, e.g., [EGNO, 7.13]. The fusion category $\mathcal{Z}(\mathcal{A})$ has a canonical braiding and there is an induction homomorphism

$$
\text { ind : } \operatorname{Aut}(\mathcal{A}) \rightarrow \operatorname{Aut}^{b r}(\mathcal{Z}(\mathcal{A})): \alpha \mapsto \operatorname{ind}(\alpha),
$$

where ind $(\alpha)(Z, \gamma)=\left(\alpha(Z), \gamma^{\alpha}\right)$ and $\gamma^{\alpha}$ is defined by the following commutative diagram

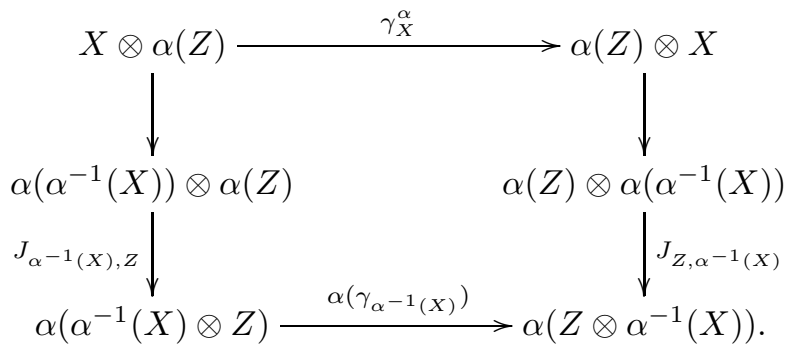

Here $\alpha^{-1}$ is a quasi-inverse of $\alpha$ and $J_{X, Y}: \alpha(X) \otimes \alpha(Z) \stackrel{\sim}{\rightarrow} \alpha(X \otimes Z)$ is the tensor functor structure of $\alpha$.

3.2. The kernel of induction. Let us recall several canonical homomorphisms associated to a fusion category $\mathcal{A}$.

Let $U(\mathcal{A})$ denote the universal grading group of $\mathcal{A}$ and let $U(\mathcal{A})_{a b}$ denote the maximal abelian quotient of $U(\mathcal{A})$. The objects $Z$ in $\mathcal{Z}(\mathcal{A})$ such that $Z=1$ as an object of $\mathcal{A}$ form an abelian group isomorphic to $\widehat{U(\mathcal{A})}$ (i.e., the group of linear characters of $U(\mathcal{A})$ ), see GN. Indeed, central object structures on $\mathbf{1}$ are in bijection with tensor automorphisms of the identity endofunctor of $\mathcal{A}$. This yields an injective homomorphism

$$
\widehat{U(\mathcal{A})} \rightarrow \operatorname{lnv}(\mathcal{Z}(\mathcal{A}))
$$


Next, the forgetful functor $F: \mathcal{Z}(\mathcal{A}) \rightarrow \mathcal{A}$ restricts to a homomorphism between groups of invertible objects:

$$
F: \operatorname{lnv}(\mathcal{Z}(\mathcal{A})) \rightarrow \operatorname{lnv}(\mathcal{A})
$$

For any invertible object $X \in \mathcal{A}$ the conjugation by $X$ is a tensor autoequivalence of $\mathcal{C}$, thus there is a group homomorphism

$$
\operatorname{conj}: \operatorname{lnv}(\mathcal{A}) \rightarrow \operatorname{Aut}(\mathcal{A}) .
$$

Proposition 3.1. The sequence of group homomorphisms

$$
0 \longrightarrow \widehat{U(\mathcal{A})} \longrightarrow \operatorname{lnv}(\mathcal{Z}(\mathcal{A})) \stackrel{F}{\longrightarrow} \operatorname{lnv}(\mathcal{A}) \stackrel{\text { conj }}{\longrightarrow} \operatorname{Aut}(\mathcal{A}) \stackrel{\text { ind }}{\longrightarrow} \operatorname{Aut}^{\text {br }}(\mathcal{Z}(\mathcal{A}))
$$

is exact.

Proof. The exactness at $\operatorname{lnv}(\mathcal{Z}(\mathcal{A}))$ is obvious. To see that the sequence is exact at $\operatorname{lnv}(\mathcal{A})$ observe that a natural tensor isomorphism between the conjugation functor

$$
V \mapsto X \otimes V \otimes X^{*}
$$

and $\operatorname{id}_{\mathcal{A}}$ is the same thing as a central structure on $X$. It remains to establish exactness at $\operatorname{Aut}(\mathcal{A})$. For $\alpha \in \operatorname{Aut}(\mathcal{A})$ let $\mathcal{A}_{\alpha}$ denote the invertible $\mathcal{A}$-bimodule category corresponding to the induced autoequivalence ind $(\alpha) \in \operatorname{Aut}^{b r}(\mathcal{Z}(\mathcal{A}))$ under isomorphism (4). This category is equivalent to the regular category $\mathcal{A}$ as a right $\mathcal{A}$-module category and left action of $\mathcal{A}$ on $\mathcal{A}_{\alpha}$ is given by

$$
(X, V) \mapsto \alpha(X) \otimes V,
$$

for all $X \in \mathcal{A}$ and $V \in \mathcal{A}_{\alpha}$, see [NR, Example 6.4]. Braided autoequivalence ind $(\alpha)$ is trivial if and only if there is an $\mathcal{A}$-bimodule equivalence between $\mathcal{A}_{\alpha}$ and $\mathcal{A}$. Such an equivalence is given by $V \mapsto X \otimes V$ for an invertible object $X$ in $\mathcal{A}$ such that $\alpha$ is the conjugation by $X$. Thus, the result follows from isomorphism (4).

Remark 3.2. The exact sequence (17) can also be obtained by combining two exact sequences from [GP, Theorem 1.3].

3.3. The image of induction. Let $\mathcal{C}$ be a non-degenerate braided fusion category and let $A$ be a Lagrangian algebra in $\mathcal{C}$. By definition [DMNO, this means that $A$ is a connected étale (i.e., commutative and separable) algebra in $\mathcal{C}$ such that $\mathrm{FPdim}(A)^{2}=\mathrm{FPdim}(\mathcal{C})$. Let $\mathcal{C}_{A}$ be the category of left $A$-modules in $\mathcal{C}$. It is a fusion category with tensor product $\otimes_{A}$.

There is a canonical braided tensor equivalence

$$
\iota_{A}: \mathcal{C} \stackrel{\sim}{\rightarrow} \mathcal{Z}\left(\mathcal{C}_{A}\right): Z \mapsto A \otimes Z .
$$

Let

$$
\operatorname{ind}_{A}: \operatorname{Aut}\left(\mathcal{C}_{A}\right) \rightarrow \operatorname{Aut}^{b r}\left(\mathcal{Z}\left(\mathcal{C}_{A}\right)\right)
$$

denote the induction homomorphism.

Theorem 3.3. Let $\alpha$ be a braided tensor autoequivalence of $\mathcal{C}$. The following condition are equivalent:

(i) there is an algebra isomorphism $\alpha(A) \cong A$,

(ii) there is $\gamma \in \operatorname{Aut}\left(\mathcal{C}_{A}\right)$ such that $\alpha=\iota_{A}^{-1} \circ \operatorname{ind}_{A}(\gamma) \circ \iota_{A}$. 
Proof. Suppose that there is an algebra isomorphism $\phi: \alpha(A) \stackrel{\sim}{\longrightarrow} A$. Define an autoequivalence $\gamma \in \operatorname{Aut}\left(\mathcal{C}_{A}\right)$ as follows. Given an $A$-module $X$ in $\mathcal{C}$ with the action $p: A \otimes X \rightarrow X$ we set $\gamma(X)=\alpha(X)$ as an object in $\mathcal{C}$, with the action

$$
A \otimes \gamma(X)=A \otimes \alpha(X) \stackrel{\phi^{-1} \otimes \operatorname{id}_{\alpha(X)}}{\longrightarrow} \alpha(A) \otimes \alpha(X) \cong \alpha(A \otimes X) \stackrel{\alpha(p)}{\longrightarrow} \alpha(X) .
$$

Then $\iota_{A} \alpha \iota_{A}^{-1}(A \otimes Z) \cong A \otimes \alpha(Z)=\gamma(A \otimes Z)$ as $A$-modules and its central structure is determined by that of $Z$. This means that $\iota_{A} \circ \alpha \circ \iota_{A}^{-1}=\operatorname{ind}_{A}(\gamma)$.

Conversely, suppose that $\alpha=\iota_{A}^{-1} \circ \operatorname{ind}_{A}(\gamma) \circ \iota_{A}$ for some $\gamma \in \operatorname{Aut}\left(\mathcal{C}_{A}\right)$. Let $F_{A}: \mathcal{Z}\left(\mathcal{C}_{A}\right) \rightarrow \mathcal{C}_{A}$ be the forgetful functor and let $I_{A}: \mathcal{C}_{A} \rightarrow \mathcal{Z}\left(\mathcal{C}_{A}\right)$ be its right adjoint. Note that $I_{A}(\mathbf{1})$ is a Lagrangian algebra in $\mathcal{Z}\left(\mathcal{C}_{A}\right)$ and $A \cong \iota_{A}^{-1}\left(I_{A}(\mathbf{1})\right)$. For any $\gamma \in \operatorname{Aut}\left(\mathcal{C}_{A}\right)$ we have a natural tensor isomorphism

$$
F_{A} \circ \operatorname{ind}(\gamma) \cong \gamma \circ F_{A} .
$$

Taking adjoints of both sides and replacing $\gamma$ by its inverse we obtain natural isomorphism ind $(\gamma) \circ I_{A} \cong I_{A} \circ \gamma$ satisfying a multiplicative property corresponding to (21) being an isomorphism of tensor functors. Applying both sides of the last isomorphism to $\mathbf{1}$ we obtain an algebra isomorphism

$$
\operatorname{ind}(\gamma)\left(I_{A}(\mathbf{1})\right) \cong I_{A}(\mathbf{1})
$$

which is equivalent to $\alpha(A) \cong A$.

Remark 3.4. It follows from Theorem 3.3 that the image of the induction homomorphism (20) is the stabilizer of (the isomorphism class of) a Lagrangian algebra $A \in \mathcal{C}$ in $\operatorname{Aut}^{b r}(\mathcal{C})$.

3.4. Orbits of the action of the Brauer-Picard group on the categorical Lagrangian Grassmannian. Let $\mathcal{A}$ be a fusion category. Denote by $\mathcal{L}(\mathcal{A})$ the groupoid of Lagrangian algebras in $\mathcal{Z}(\mathcal{A})$. It is known that $\mathcal{L}(\mathcal{A})$ is equivalent to the groupoid of indecomposable $\mathcal{A}$-module categories via $A \mapsto \mathcal{Z}(\mathcal{A})_{A}$. The group $\operatorname{Aut}^{b r}(\mathcal{Z}(\mathcal{A})$ ) (i.e., the Brauer-Picard group of $\mathcal{A}$ ) acts on $\mathcal{L}(\mathcal{A})$ in an obvious way.

Remark 3.5. The groupoid $\mathcal{L}(\mathcal{A})$ can be thought of as a categorical analogue of a Lagrangian Grassmannian. In $\left[\mathrm{NR}\right.$, the action of $\operatorname{Aut}^{b r}(\mathcal{Z}(\mathcal{A}))$ on the subset of $\mathcal{L}(\mathcal{A})$ consisting of algebras coming from Lagrangian subcategories of $\mathcal{Z}(\mathcal{A})$ was considered.

The following result extends Theorem 3.3 from the previous section.

Proposition 3.6. Let $A$ and $B$ be Lagrangian algebras in $\mathcal{Z}(\mathcal{A})$. There exists a tensor equivalence $\mathcal{Z}(\mathcal{A})_{A} \cong \mathcal{Z}(\mathcal{A})_{B}$ if and only if there exists $\alpha \in \operatorname{Aut}^{b r}(\mathcal{Z}(\mathcal{A})$ ) such that $\alpha(A) \cong B$ as algebras in $\mathcal{Z}(\mathcal{A})$.

Proof. Suppose there is a tensor equivalence $\phi: \mathcal{Z}(\mathcal{A})_{A} \rightarrow \mathcal{Z}(\mathcal{A})_{B}$. Let ind $(\phi)$ : $\mathcal{Z}\left(\mathcal{Z}(\mathcal{A})_{A}\right) \rightarrow \mathcal{Z}\left(\mathcal{Z}(\mathcal{A})_{B}\right)$ be the induced braided tensor equivalence and let

$$
\iota_{A}: \mathcal{Z}(\mathcal{A}) \rightarrow \mathcal{Z}\left(\mathcal{Z}(\mathcal{A})_{A}\right), \quad \iota_{B}: \mathcal{Z}(\mathcal{B}) \rightarrow \mathcal{Z}\left(\mathcal{Z}(\mathcal{A})_{B}\right)
$$

be braided equivalences defined in the previous section. Then $\alpha=\iota_{B}^{-1} \circ \operatorname{ind}(\phi) \circ \iota_{A}$ is a braided autoequivalence of $\mathcal{Z}(\mathcal{A})$ that maps $A$ to $B$ (the verification of this fact is completely parallel to one in the proof of Theorem 3.3 .

Conversely, if $\alpha \in \operatorname{Aut}^{b r}(\mathcal{Z}(\mathcal{A}))$ then there is a tensor equivalence

$$
\mathcal{Z}(\mathcal{A})_{A} \cong \mathcal{Z}(\mathcal{A})_{\alpha(A)}
$$

given by $X \mapsto \alpha(X)$ for all objects $X \in \mathcal{Z}(\mathcal{A})_{A}$. 
Remark 3.7. Proposition 3.6 implies that orbits of the action of $\operatorname{Aut}^{b r}(\mathcal{Z}(\mathcal{A}))$ on the set of isomorphism classes of Lagrangian algebras in $\mathcal{Z}(\mathcal{A})$ are parameterized by equivalence classes of fusion categories categorically Morita equivalent to $\mathcal{A}$.

Equivalently, we have a parameterization of orbits of the action of $\operatorname{BrPic}(\mathcal{A})$ on the set of equivalence classes of indecomposable left $\mathcal{A}$-module categories. Namely, two $\mathcal{A}$-module categories belong to the same orbit if and only if the corresponding dual fusion categories are equivalent. A related result is established by Galindo and Plavnik in [GP, Theorem 1.4].

\section{Representation CAtegories of twisted Group Doubles}

Let $G$ be a finite group and let $\omega \in H^{3}\left(G, k^{\times}\right)$.

4.1. Invertible objects of the center of a pointed fusion category. Let $Z(G)$ denote the center of $G$. For any $a \in Z(G)$ let

$$
\beta_{a}(x, y)=\frac{\omega(a, x, y) \omega(x, y, a)}{\omega(x, a, y)}, \quad x, y \in G .
$$

It is known that $\beta_{a}$ is a 2 -cocycle and that the map

$$
\beta: Z(G) \rightarrow H^{2}\left(G, k^{\times}\right): a \mapsto \beta_{a}
$$

is a group homomorphism.

The invertible objects of $\mathcal{Z}(\mathcal{C}(G, \omega))$ are well known, see, e.g., [DPR. The exact sequence (24) in the next proposition can be found in [GP, Example 6.2]. We include its proof for the sake of completeness.

For any group $G$ let $\widehat{G}$ denote the group of linear characters of $G$.

Proposition 4.1. The following sequence

$$
0 \rightarrow \widehat{G} \rightarrow \operatorname{lnv}\left(\mathcal{Z}(\mathcal{C}(G, \omega)) \stackrel{F}{\rightarrow} Z(G) \stackrel{\beta}{\rightarrow} H^{2}\left(G, k^{\times}\right)\right.
$$

is exact. Here $F$ is given by the forgetful functor.

Proof. The central structures on the identity object of $\mathcal{C}(G, \omega)$ are parameterized by linear characters of $G$. It is clear that in order to have a central structure the invertible object of $\mathcal{C}(G, \omega)$ must correspond to an element of $Z(G)$. Finally, it follows from (22) that $a \in Z(G)$ admits a central structure if and only if $\beta_{a}$ is a coboundary.

Remark 4.2. Proposition 4.1 appears (in a different form) in MN2, Proposition 5.2]. It can also be derived from the exact sequence (17).

Corollary 4.3. The category $\mathcal{Z}(\mathcal{C}(G, \omega))$ is pointed if and only if $G$ is abelian and $\beta$ is zero.

Corollary 4.4. Suppose that $G$ is abelian. Then $\mathcal{Z}(\mathcal{C}(G, \omega))_{p t}$ is Lagrangian if and only if $\beta$ is injective.

Proof. Note that $\mathcal{Z}(\mathcal{C}(G, \omega))_{p t}$ contains the Lagrangian subcategory $\mathcal{L}=\operatorname{Rep}(G)$ consisting of central objects supported on $\mathbf{1}$. Clearly, $\mathcal{Z}(\mathcal{C}(G, \omega))_{p t}=\mathcal{L}$ if and only if the forgetful homomorphism $F: \operatorname{lnv}(\mathcal{Z}(\mathcal{C})) \rightarrow \operatorname{Inv}(\mathcal{C})$ is trivial, i.e., if and only if $\beta$ is injective. 
4.2. Image of induction in the pointed case. Let $G$ be a finite group and let $\omega \in H^{3}\left(G, k^{\times}\right)$.

Let OutStab $(\omega)=\operatorname{Stab}(\omega) / \operatorname{Inn}(G)$ denote the subgroup of Out $(G)$ consisting of classes of automorphisms $a$ such that $\omega \circ(a \times a \times a)$ is cohomologous to $\omega$.

Let $I(G, \omega)$ be the image of induction $\operatorname{Aut}(\mathcal{C}(G, \omega)) \rightarrow \operatorname{Aut}^{b r}(\mathcal{Z}(\mathcal{C}(G, \omega)))$.

Proposition 4.5. There is an exact sequence

$$
Z(G) \stackrel{\beta}{\rightarrow} H^{2}\left(G, k^{\times}\right) \stackrel{\text { ind }}{\longrightarrow} I(G, \omega) \rightarrow \operatorname{OutStab}(\omega) \rightarrow 0
$$

Proof. Using the exact sequence (17) we see that

$$
I(G, \omega) \cong \operatorname{Aut}(\mathcal{C}(G, \omega)) / \operatorname{Ker}(\text { ind }) \cong \operatorname{Aut}(\mathcal{C}(G, \omega)) / \operatorname{Im}(\text { conj }),
$$

where conj is given by (16). The image of conj in $\operatorname{Aut}(\mathcal{C}(G, \omega))$ is generated by $\operatorname{lnn}(G)=G / Z(G)$ and the image of $\beta: Z(G) \rightarrow H^{2}\left(G, k^{\times}\right) \subset$ Aut $(\mathcal{C}(G, \omega))$ defined in (23) (note that the conjugation by $a \in Z(G)$ gives rise to a non-trivial tensor autoequivalence of $\mathcal{C}(G, \omega)$ precisely when $\beta_{a}$ is non-trivial in $\left.H^{2}\left(G, k^{\times}\right)\right)$. By (8), this implies the statement.

Corollary 4.6. We have an exact sequence

$0 \rightarrow \widehat{G} \rightarrow \operatorname{Inv}\left(\mathcal{Z}(\mathcal{C}(G, \omega)) \stackrel{F}{\rightarrow} Z(G) \stackrel{\beta}{\rightarrow} H^{2}\left(G, k^{\times}\right) \stackrel{\text { ind }}{\rightarrow} I(G, \omega) \rightarrow \operatorname{OutStab}(\omega) \rightarrow 0\right.$.

Corollary 4.7. Let $\mathcal{C}$ be a non-degenerate braided fusion category and let $\mathcal{L}=$ $\operatorname{Rep}(G)$ be a Lagrangian subcategory of $\mathcal{C}$ such that $\alpha(\mathcal{L}) \cong \mathcal{L}$ for every $\alpha \in \operatorname{Aut}^{\text {br }}(\mathcal{C})$. Then $\mathcal{C} \cong \mathcal{Z}(\mathcal{C}(G, \omega))$ for some $\omega \in H^{3}\left(G, k^{\times}\right)$and the induction homomorphism

$$
\operatorname{Aut}(\mathcal{C}(G, \omega)) \rightarrow \operatorname{Aut}^{b r}(\mathcal{Z}(\mathcal{C}(G, \omega))
$$

is surjective, i.e., $\operatorname{Aut}^{b r}(\mathcal{Z}(\mathcal{C}(G, \omega))=I(G, \omega)$.

Proof. This follows from Theorem 3.3 and Proposition 4.5 .

4.3. Braided equivalences between representation categories of twisted group doubles. Let $G$ be a finite group and let $A \subset G$ be a normal abelian subgroup. Let $K:=G / A$ be the quotient group, so that there is an extension

$$
0 \rightarrow A \rightarrow G \rightarrow K \rightarrow 0 \text {. }
$$

Such an extension is determined up to an isomorphism by the action of $K$ on $G$ (denoted by $(x, a) \mapsto x \cdot a$ for $x \in K, a \in A$ ) and the cohomology class of a 2-cocycle $\kappa \in Z^{2}(K, A)$, so that elements of $G$ are identified with pairs $(a, x) \in A \times K$ and the multiplication is given by

$$
(a, x)(b, y)=(a(x \cdot b) \kappa(x, y), x y), \quad a, b \in A, x, y \in K .
$$

It was shown in [MN1, $\mathbb{N}$ ] that the fusion category $\operatorname{Vec}_{G}$ is categorically Morita equivalent to $\operatorname{Vec}_{\widehat{A} \rtimes K}^{\omega}$, where $\widehat{A}$ is the dual of the $K$-module $A$ and the 3 -cocycle $\omega \in Z^{3}\left(\widehat{A} \rtimes K, k^{\times}\right)$is defined by

$$
\omega\left(\left(\rho_{1}, x_{1}\right),\left(\rho_{2}, x_{2}\right),\left(\rho_{3}, x_{3}\right)\right)=\rho_{1}\left(\kappa\left(x_{2}, x_{3}\right)\right),
$$

for all $\rho_{1}, \rho_{2}, \rho_{3} \in \widehat{A}$ and $x_{1}, x_{2}, x_{3} \in K$. 
Remark 4.8. In fact, in [MN1, N, U] all pairs of Morita equivalent pointed fusion categories (i.e., all pairs of of twisted group doubles with braided tensor equivalent representation categories) were classified. In this paper we will only use the special case described above.

Thus, there exists a braided equivalence

$$
\mathcal{Z}\left(\operatorname{Vec}_{G}\right) \cong \mathcal{Z}\left(\operatorname{Vec}_{\widehat{A} \rtimes K}^{\omega}\right) .
$$

So for computational purposes the double of $G$ can be replaced by the twisted double of an easier group $\widehat{A} \rtimes K$.

\section{TWisted DOUbles OF ELEMENTARY ABELIAN $p$-GROUPS AND THEIR BRAUER-PICARD GROUPS}

Let $p$ be a prime, let $n$ be a positive integer, and let $V_{n}$ denote the elementary abelian $p$-group of order $p^{n}$. We have Aut $\left(V_{n}\right)=G L_{n}\left(\mathbb{F}_{p}\right)$. Below we will also view $V_{n}$ as an $n$-dimensional vector space over the field $\mathbb{F}_{p}$ with $p$ elements. We will denote $V_{n}^{*}=\operatorname{Hom}\left(V_{n}, \mathbb{F}_{p}\right)$ the dual vector space.

As before, for $\omega \in H^{3}\left(V_{n}, k^{\times}\right)$we denote $\mathcal{C}\left(V_{n}, \omega\right)$ the category of $V_{n}$-graded vector spaces with the associativity constraint twisted by $\omega$.

For a vector space $W$ we denote

$$
\bigwedge(W)=\bigoplus_{i=0}^{\infty} \bigwedge^{i}(W) \text { and } \mathrm{S}(W)=\bigoplus_{i=0}^{\infty} \mathrm{S}^{i}(W)
$$

the alternating and symmetric algebras of $W$. When $W$ has a basis $\left\{w_{1}, \ldots, w_{n}\right\}$ we also write $\Lambda(W)=\bigwedge\left(w_{1}, \ldots, w_{n}\right)$ and $\mathbf{S}(W)=\mathbf{S}\left(w_{1}, \ldots, w_{n}\right)$.

5.1. The Brauer-Picard group of $\mathcal{C}\left(V_{n}, \omega\right)$ when $p$ is odd. Let $p$ be an odd prime.

The cohomology ring $H^{\bullet}\left(V_{n}, \mathbb{F}_{p}\right)$ is well known (see, e.g., $[\mathrm{A}$ ), namely

$$
H^{\bullet}\left(V_{n}, \mathbb{F}_{p}\right)=\bigwedge\left(x_{1}, \ldots, x_{n}\right) \otimes_{\mathbb{F}_{p}} \mathbb{F}_{p}\left[y_{1}, \ldots, y_{n}\right],
$$

where $\operatorname{deg}\left(x_{i}\right)=1$ and $\operatorname{deg}\left(y_{i}\right)=2$ for all $i=1, \ldots, n$.

The cocycles representing generators $x_{i}$ and $y_{i}$ can be explicitly described as follows (below we identify cocycles with the cohomology classes they represent). Define $x_{i}: V_{n} \rightarrow \mathbb{F}_{p}$ and $y_{i}: V_{n} \times V_{n} \rightarrow \mathbb{F}_{p}$ by

$$
x_{i}(v)=v_{i}
$$

and

$$
y_{i}(u, v)= \begin{cases}0 & \text { if } u_{i}+v_{i}<p \\ 1 & \text { if } u_{i}+v_{i} \geq p\end{cases}
$$

for all $v=\left(v_{1}, \ldots v_{n}\right), u=\left(u_{1}, \ldots u_{n}\right)$ in $V_{n}$ and $i=1, \ldots n$. Here we view $u_{i}, v_{i}$ as elements of $\{0,1, \ldots, p-1\}$ and add them as usual integers.

In particular,

$$
H^{3}\left(V_{n}, \mathbb{F}_{p}\right)=\bigwedge^{3}\left(x_{1}, \ldots, x_{n}\right) \bigoplus \mathbb{F}_{p}\left\langle x_{i} \cup y_{j} \mid i, j=1, \ldots, n\right\rangle,
$$

where $\cup$ denotes the cup product. Note that the second summand in (33) is isomorphic to $V_{n}^{*} \otimes V_{n}^{*}$ as a $G L_{n}\left(\mathbb{F}_{p}\right)$-module. 
Proposition 5.1. There is an isomorphism of $G L_{n}\left(\mathbb{F}_{p}\right)$-modules:

$$
H^{3}\left(V_{n}, k^{\times}\right) \cong \bigwedge^{3}\left(V_{n}^{*}\right) \bigoplus \mathrm{S}^{2}\left(V_{n}^{*}\right) .
$$

Proof. The automorphism of $k^{\times}$given by $\xi \mapsto \xi^{p}$ yields an exact sequence of abelian groups:

$$
0 \longrightarrow \mathbb{F}_{p} \longrightarrow k^{\times} \longrightarrow k^{\times} \longrightarrow
$$

where $\mathbb{F}_{p}$ is identified with the group of $p$ th roots of 1 in $k$. This, by functoriality, yields a long exact sequence of $G L_{n}\left(\mathbb{F}_{p}\right)$-modules:

$$
\begin{aligned}
& \cdots \longrightarrow H^{m-1}\left(V_{n}, \mathbb{F}_{p}\right) \longrightarrow H^{m-1}\left(V_{n}, k^{\times}\right) \stackrel{0}{\longrightarrow} H^{m-1}\left(V_{n}, k^{\times}\right) \\
& \longrightarrow H^{m}\left(V_{n}, \mathbb{F}_{p}\right) \longrightarrow H^{m}\left(V_{n}, k^{\times}\right) \stackrel{0}{\longrightarrow} H^{m}\left(V_{n}, k^{\times}\right) \longrightarrow \cdots
\end{aligned}
$$

Note that the map $H^{m-1}\left(V_{n}, k^{\times}\right) \rightarrow H^{m-1}\left(V_{n}, k^{\times}\right)$induced by taking the $p$ th power is zero since the exponent of $H^{m}\left(V_{n}, k^{\times}\right)$is $p$. The latter fact follows from isomorphism $H^{m}\left(V_{n}, k^{\times}\right)=H^{m+1}\left(V_{n}, \mathbb{Z}\right)$ and the Künneth formula for the direct product in cohomology.

In particular, there is a short exact sequence of $G L_{n}\left(\mathbb{F}_{p}\right)$-modules

$$
0 \rightarrow H^{2}\left(V_{n}, k^{\times}\right) \stackrel{\delta}{\rightarrow} H^{3}\left(V_{n}, \mathbb{F}_{p}\right) \rightarrow H^{3}\left(V_{n}, k^{\times}\right) \rightarrow 0 .
$$

We claim that the image of inclusion

$$
\delta: H^{2}\left(V_{n}, k^{\times}\right)=\bigwedge^{2}\left(V_{n}^{*}\right) \rightarrow H^{3}\left(V_{n}, \mathbb{F}_{p}\right)
$$

is the subspace of $\mathbb{F}_{p}\left\langle x_{i} \cup y_{j} \mid i, j=1, \ldots, n\right\rangle \subset H^{3}\left(V_{n}, \mathbb{F}_{p}\right)$ consisting of cohomology classes of the form $\sum_{i, j=1}^{n} a_{i j} x_{i} \cup y_{j}$, where $A=\left\{a_{i j}\right\}$ is an anti-symmetric matrix over $\mathbb{F}_{p}$.

Indeed, (36) is given by the connecting homomorphism which is explicitly computed as follows. Fix a primitive $p$-th root of unity $\xi$ in $k$ and consider homomorphism

$$
\mathbb{F}_{p} \rightarrow k^{\times}: a \mapsto \xi^{a} .
$$

A class in $H^{2}\left(V_{n}, k^{\times}\right)$is represented by a 2 -cocycle $\mu: V_{n} \times V_{n} \rightarrow k^{\times}$given by

$$
\mu(u, v)=\xi^{(A u, v)}, \quad u, v \in V_{n},
$$

where $A=\left\{a_{i j}\right\}$ is an $n$-by- $n$ matrix over $\mathbb{F}_{p}$ and $(A u, v)=\sum_{i, j}^{n} a_{i j} u_{i} v_{j}$ is viewed as a non-negative integer.

Let $\lambda$ be a fixed $p$ th root of $\xi$ in $k$. Take a 2-cochain $\nu: V_{n} \times V_{n} \rightarrow k^{\times}$defined by

$$
\nu(u, v)=\lambda^{(A u, v)}, \quad u, v \in V_{n} .
$$

For non-negative integers $a, b$ let us denote $\{a, b\}$ the integral part of $\frac{a+b}{p}$. We have

$$
\begin{aligned}
& \nu(u, v) \nu\left(u^{\prime}, v\right)=\nu\left(u+u^{\prime}, v\right) \xi^{\left\{(A u, v),\left(A u^{\prime}, v\right)\right\}}, \\
& \nu(u, v) \nu\left(u, v^{\prime}\right)=\nu\left(u, v+v^{\prime}\right) \xi^{\left\{(A u, v),\left(A u, v^{\prime}\right)\right\}},
\end{aligned}
$$

for all $u, u^{\prime}, v, v^{\prime} \in V_{n}$. Using these identities we compute the differential of $\nu$ :

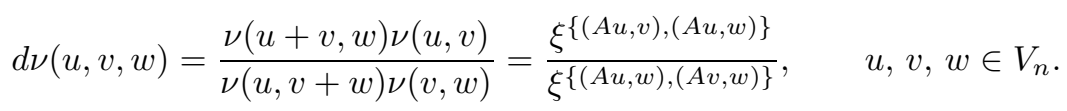


Fix $k, l \in\{1,2, \ldots, n\}$ and take $A$ such that $a_{i j}=1$ if $i=k$ and $j=l$ and $a_{i j}=0$ otherwise. Then the previous calculation yields

$$
d \nu(u, v, w)=\frac{\xi^{u_{k}\left\{v_{l}, w_{l}\right\}}}{\xi^{w_{l}\left\{u_{k}, v_{k}\right\}}} .
$$

Since $d \nu(u, v, w)=\xi^{\delta(\mu)(u, v, w)}$, we conclude that in this case

$$
\delta(\mu)(u, v, w)=u_{k}\left\{v_{l}, w_{l}\right\}-w_{l}\left\{u_{k}, v_{k}\right\} .
$$

Comparing this with (31) and (32) (note that $y_{i}(u, v)=\left\{u_{i}, v_{i}\right\}$ ) we conclude that the image of $\delta$ is spanned by

$$
x_{k} \cup y_{l}-x_{l} \cup y_{k}, k, l=1, \ldots, n,
$$

as claimed. The quotient of $V_{n}^{*} \otimes V_{n}^{*}$ by this space is isomorphic to $\mathrm{S}^{2}\left(V_{n}^{*}\right)$ via the symmetrization map. Since

$$
H^{3}\left(V_{n}, k^{\times}\right)=H^{3}\left(V_{n}, \mathbb{F}_{p}\right) / \operatorname{Image}\left(\delta: H^{2}\left(V_{n}, k^{\times}\right) \rightarrow H^{3}\left(V_{n}, \mathbb{F}_{p}\right)\right),
$$

the statement follows from the last claim and (33).

Given a cohomology class $\omega \in H^{3}\left(V_{n}, k^{\times}\right)$denote

$$
\omega=\omega_{\text {alt }}+\omega_{\text {sym }}, \quad \omega_{\text {alt }} \in \bigwedge^{3}\left(V_{n}^{*}\right), \quad \omega_{\text {sym }} \in \mathrm{S}^{2}\left(V_{n}^{*}\right)
$$

the decomposition of $\omega$ from (34).

Let $V$ be a vector space. Consider the interior derivation

$$
\iota: V \otimes \bigwedge^{3}\left(V^{*}\right) \rightarrow \bigwedge^{2}\left(V^{*}\right): v \otimes \phi \mapsto \iota_{v}(\phi),
$$

given by

$$
\iota_{v}(x \wedge y \wedge z)=\langle v, z\rangle x \wedge y-\langle v, y\rangle x \wedge z+\langle v, x\rangle y \wedge z,
$$

for all $v \in V, x, y, z \in V^{*}$, and extended to $\bigwedge^{3}\left(V^{*}\right)$ by linearity.

The radical of $\phi \in \Lambda^{3}\left(V^{*}\right)$ is defined as

$$
\operatorname{Rad}(\phi):=\left\{u \in V \mid \iota_{u}(\phi)=0\right\} .
$$

We say that $\phi \in \bigwedge^{3}\left(V^{*}\right)$ is non-degenerate if $\operatorname{Rad}(\phi)=0$.

Proposition 5.2. Let $v \in V_{n}$ and let $\omega \in H^{3}\left(V_{n}, k^{\times}\right)$. Then $v$, regarded as a simple object in $\mathcal{C}\left(V_{n}, \omega\right)$, is in the image of $\operatorname{Inv}\left(\mathcal{Z}\left(\mathcal{C}\left(V_{n}, \omega\right)\right)\right) \rightarrow \operatorname{Inv}\left(\mathcal{C}\left(V_{n}, \omega\right)\right)$ if and only if $v \in \operatorname{Rad}\left(\omega_{\text {alt }}\right)$.

Proof. We claim that in this case the homomorphism

$$
\beta: V_{n} \rightarrow H^{2}\left(V_{n}, k^{\times}\right)=\bigwedge^{2}\left(V_{n}^{*}\right)
$$

defined by (22) and (23) is given by

$$
\beta(v)=\iota_{v}\left(\omega_{\text {alt }}\right), \quad v \in V_{n} .
$$

Let us first prove this claim when $\omega=\omega_{\text {alt }} \in \Lambda^{3}\left(V_{n}^{*}\right)$ (i.e., when the symmetric part of $\omega$ in (38) is trivial). Such an $\omega$ is a linear combination of 3-cocycles $\omega_{i j k}$ $(i, j, k$ are distinct elements of $\{1, \ldots, n\})$, given by $\omega(u, v, w)=\xi^{u_{i} v_{j} w_{k}}$, where $\xi$ is a fixed primitive $p$ th root of 1 in $k$. So we may assume that $\omega=\omega_{i j k}$. We have

$$
\beta(v)=v_{i} x_{j} \wedge x_{k}-v_{j} x_{i} \wedge x_{k}+v_{k} x_{i} \wedge x_{j}, \quad v=\left(v_{1}, \ldots, v_{n}\right) \in V_{n},
$$

where $x_{i}$ are defined by (31). Thus, $\beta(v)=\iota_{v}\left(\omega_{i j k}\right)$ and (40) is true in this case. 
Next, let us prove the claim when $\omega=\omega_{\text {sym }} \in \mathrm{S}^{2}\left(V_{n}^{*}\right)$. We need to check that $\beta=0 \in H^{2}\left(V_{n}, k^{\times}\right)$in this case. We may assume that $\omega$ is the image of $x_{i} \cup y_{j} \in H^{3}\left(V_{n}, \mathbb{F}_{p}\right)$ under (35) i.e.,

$$
\omega(u, v, w)=\xi^{x_{i}(u) y_{j}(v, w)}, \quad u, v, w \in V_{n} .
$$

Since $y_{j}$ is symmetric (see (32)) we conclude that $\beta(v)$ is the image of $v_{i} y_{j}$ under the homomorphism $H^{2}\left(V_{n}, \mathbb{F}_{p}\right) \rightarrow H^{2}\left(V_{n}, k^{\times}\right)$and, hence, is trivial.

So, (40) is true for all $\omega \in H^{3}\left(V_{n}, k^{\times}\right)$and the result follows from Proposition 4.1

Corollary 5.3. The category $\mathcal{Z}\left(\mathcal{C}\left(V_{n}, \omega\right)\right)$ is pointed if and only if $\omega_{\text {alt }}=0$.

Corollary 5.4. $\mathcal{Z}\left(\mathcal{C}\left(V_{n}, \omega\right)\right)_{p t}=\operatorname{Rep}\left(V_{n}\right)$ if and only if $\omega_{\text {alt }}$ is non-degenerate. In this case $\mathcal{Z}\left(\mathcal{C}\left(V_{n}, \omega\right)\right)_{p t}$ is the trivial component of the universal grading of $\mathcal{Z}\left(\mathcal{C}\left(V_{n}, \omega\right)\right)$ and the universal grading group of $\mathcal{Z}\left(\mathcal{C}\left(V_{n}, \omega\right)\right)$ is $\operatorname{Hom}\left(V_{n}, k^{\times}\right)$, the dual group of $V_{n}$.

Proof. This follows from Corollary 4.4

Theorem 5.5. Let $\omega \in H^{3}\left(V_{n}, k^{\times}\right)$be such that $\omega_{\text {alt }}$ is non-degenerate. There is an exact sequence of groups:

$$
0 \rightarrow V_{n} \stackrel{\iota\left(\omega_{a l t}\right)}{\longrightarrow} \bigwedge^{2}\left(V_{n}^{*}\right) \rightarrow \operatorname{BrPic}\left(\mathcal{C}\left(V_{n}, \omega\right)\right) \rightarrow \operatorname{Stab}_{V_{n}}(\omega) \rightarrow 0 .
$$

Proof. This follows from Proposition 4.5 and Corollary 4.7 applied to $G=V_{n}$.

Theorem 5.5 implies that the Brauer-Picard group of $\mathcal{C}\left(V_{n}, \omega\right)$ is an extension of $\operatorname{Stab}_{V_{n}}(\omega)=\operatorname{Stab}\left(\omega_{\text {alt }}\right) \cap \operatorname{Stab}\left(\omega_{\text {sym }}\right)$ by an elementary abelian $p$-group.

5.2. The Brauer-Picard group of $\mathcal{C}\left(V_{n}, \omega\right)$ when $p=2$. It is known (see, e.g., (A]) that

$$
H^{\bullet}\left(V_{n}, \mathbb{F}_{2}\right)=\mathbb{F}_{2}\left[x_{1}, \ldots, x_{n}\right],
$$

where $x_{i}, i=1, \ldots, n$ are one-dimensional generators represented by 1-cocycles

$$
x_{i}(v)=v_{i}, \quad \text { where } v=\left(v_{1}, \ldots v_{n}\right) \in V_{n} .
$$

Proposition 5.6. There is an isomorphism of $G L_{n}\left(\mathbb{F}_{2}\right)$-modules:

$$
H^{3}\left(V_{n}, k^{\times}\right) \cong \mathrm{S}^{3}\left(x_{1}, \ldots, x_{n}\right) / \mathbb{F}_{2}\left\langle x_{i}^{2} x_{j}+x_{i} x_{j}^{2} \mid i, j=1, \ldots, n\right\rangle .
$$

Proof. The argument is similar to that of Proposition 5.1. There is a short exact sequence of $G L_{n}\left(\mathbb{F}_{2}\right)$-modules

$$
0 \rightarrow H^{2}\left(V_{n}, k^{\times}\right) \rightarrow H^{3}\left(V_{n}, \mathbb{F}_{2}\right) \rightarrow H^{3}\left(V_{n}, k^{\times}\right) \rightarrow 0 .
$$

The same computation as in the proof of Proposition 5.1 shows image of inclusion of $H^{2}\left(V_{n}, k^{\times}\right)=\bigwedge^{2}\left(V_{n}^{*}\right)$ into $H^{3}\left(V_{n}, \mathbb{F}_{p}\right)=\mathrm{S}^{3}\left(x_{1}, \ldots, x_{n}\right)$ is spanned by elements $x_{k} \cup y_{l}+x_{l} \cup y_{k}$ with $k, l=1, \ldots, n$ (see (37)). Since for $p=2$ we have $y_{k}=x_{k}^{2}$, the result follows.

Proposition 5.7. There is a short exact sequence of $G L_{n}\left(\mathbb{F}_{2}\right)$-modules:

$$
0 \rightarrow \mathrm{S}^{2}\left(V_{n}^{*}\right) \rightarrow H^{3}\left(V_{n}, k^{\times}\right) \stackrel{\pi}{\rightarrow} \bigwedge^{3}\left(V_{n}^{*}\right) \rightarrow 0 .
$$


Proof. Using Proposition 5.6 we see that $H^{3}\left(V_{n}, k^{\times}\right)$contains a $G L_{n}\left(\mathbb{F}_{2}\right)$-submodule spanned by $x_{i}^{2} x_{j}, i, j=1, \ldots, n$ (modulo $\mathbb{F}_{2}\left\langle x_{i}^{2} x_{j}+x_{i} x_{j}^{2} \mid i, j=1, \ldots, n\right\rangle$ ). Clearly, this submodule is isomorphic to $\mathrm{S}^{2}\left(V_{n}^{*}\right)$ and the corresponding quotient is isomorphic to $\bigwedge^{3}\left(V_{n}^{*}\right)$ (the cosets are represented by polynomials $x_{i} x_{j} x_{k}$, where $i, j, k=1, \ldots, n$ are distinct).

For $\omega \in H^{3}\left(V_{n}, k^{\times}\right)$let

$$
\omega_{\text {alt }}=\pi(\omega) \in \bigwedge^{3}\left(V_{n}^{*}\right) .
$$

Proposition 5.8. Let $v \in V_{n}$ and let $\omega \in H^{3}\left(V_{n}, k^{\times}\right)$. Then $v$, regarded as a simple object in $\mathcal{C}\left(V_{n}, \omega\right)$, is in the image of $\operatorname{Inv}\left(\mathcal{Z}\left(\mathcal{C}\left(V_{n}, \omega\right)\right)\right) \rightarrow \operatorname{lnv}\left(\mathcal{C}\left(V_{n}, \omega\right)\right)$ if and only if $v \in \operatorname{Rad}\left(\omega_{\text {alt }}\right)$.

Proof. This is similar to proof of Proposition 5.2

Corollary 5.9. The category $\mathcal{Z}\left(\mathcal{C}\left(V_{n}, \omega\right)\right)$ is pointed if and only if $\omega_{\text {alt }}=0$.

Corollary 5.10. Corollary 5.4 and Theorem 5.5 hold for $p=2$ (but note that the meaning of $\omega_{\text {alt }}$ is different in this case, $c f$. (45))

Remark 5.11. Representation categories of twisted group doubles of elementary abelian 2-groups and braided tensor equivalences between them were studied by Goff, Mason, and Ng in GMN. The above results about the third cohomology of $V_{n}$ and invertible objects of $\mathcal{Z}\left(\mathcal{C}\left(V_{n}, \omega\right)\right)$ can be derived from that paper.

\section{Computing Brauer-Picard groups: examples}

Let $p$ be a prime. A $p$-group $G$ is called extra special if its center $Z$ is cyclic of order $p$ and $G / Z$ is elementary abelian. Such groups are well known: for each positive integer $n$ there exist precisely two non-isomorphic extra special $p$-groups of order $p^{2 n+1}$.

Since $G$ is a central extension of the form

$$
0 \rightarrow \mathbb{F}_{p} \rightarrow G \rightarrow V_{2 n} \rightarrow 0,
$$

corresponding to some cohomology class $\kappa_{G} \in H^{2}\left(V_{n}, \mathbb{F}_{p}\right)$ we can apply the results of Section 4.3. Namely, there is a braided equivalence

$$
\mathcal{Z}(\mathcal{C}(G, 1)) \cong \mathcal{Z}\left(\mathcal{C}\left(V_{2 n+1}, \omega_{G}\right)\right),
$$

where $\omega_{G} \in H^{3}\left(G, k^{\times}\right)$is obtained from $\kappa_{G}$ as follows. Choose a generator $x_{0}$ of $H^{1}\left(\mathbb{F}_{p}, \mathbb{F}_{p}\right)=\mathbb{F}_{p}^{*}$ and consider the cup product

$$
H^{1}\left(\mathbb{F}_{p}, \mathbb{F}_{p}\right) \otimes_{\mathbb{F}_{p}} H^{2}\left(V_{n}, \mathbb{F}_{p}\right) \rightarrow H^{3}\left(V_{2 n+1}, \mathbb{F}_{p}\right): x_{0} \otimes \kappa \mapsto x_{0} \cup \kappa .
$$

Proposition 6.1. $\omega_{G}$ is the image of $x_{0} \cup \kappa_{G}$ under the projection

$$
H^{3}\left(V_{2 n+1}, \mathbb{F}_{p}\right) \rightarrow H^{3}\left(V_{2 n+1}, k^{\times}\right) .
$$

Proof. This follows from (29). 
6.1. Brauer-Picard groups of representation categories of extra special $p$-groups for odd $p$. Let $p$ be an odd prime and let $n$ be a positive integer. Let $D$ and $Q$ denote extra special groups of order $p^{2 n+1}$ and exponents $p$ and $p^{2}$ respectively. They can be constructed as follows. Let $M=(\mathbb{Z} / p \mathbb{Z} \times \mathbb{Z} / p \mathbb{Z}) \rtimes \mathbb{Z} / p \mathbb{Z}$ and $N=\mathbb{Z} / p^{2} \mathbb{Z} \rtimes \mathbb{Z} / p \mathbb{Z}$ be non-abelian groups of order $p^{3}$. Then $D$ is the central product of $n$ copies of $M$ and $Q$ is the central product of $N$ and $n-1$ copies of $M$.

It is straightforward to compute cohomology classes $\kappa_{D}, \kappa_{Q} \in H^{2}\left(V_{2 n}, \mathbb{F}_{p}\right)$ corresponding to extension (46) with $G=D, Q$. We have

$$
\begin{aligned}
\kappa_{D} & =\sum_{i=1}^{n} x_{2 i-1} x_{2 i}, \\
\kappa_{Q} & =\sum_{i=1}^{n} x_{2 i-1} x_{2 i}+y_{1},
\end{aligned}
$$

where generators $x_{i}, y_{i}$ are defined by (31) and (32).

Recall from Proposition 5.1 that there is a $G L_{2 n+1}\left(\mathbb{F}_{p}\right)$-module isomorphism

$$
H^{3}\left(V_{2 n+1}, k^{\times}\right) \cong \bigwedge^{3}\left(x_{0}, x_{1}, \ldots, x_{2 n}\right) \oplus \mathrm{S}^{2}\left(z_{0}, z_{1}, \ldots, z_{2 n}\right),
$$

hence elements $\omega \in H^{3}\left(V_{2 n+1}, k^{\times}\right)$correspond to pairs $\left(\omega_{\text {alt }}, \omega_{\text {sym }}\right)$, where $\omega_{\text {alt }}$ is a degree 3 element of the exterior algebra and $\omega_{\text {sym }}$ is a degree 2 element of the symmetric algebra.

Below we identify $\omega$ with its image under isomorphism (50).

Proposition 6.2. We have

$$
\begin{aligned}
& \omega_{D}=\left(x_{0} \wedge\left(\sum_{i=1}^{n} x_{2 i-1} \wedge x_{2 i},\right), 0\right) \\
& \omega_{Q}=\left(x_{0} \wedge\left(\sum_{i=1}^{n} x_{2 i-1} \wedge x_{2 i},\right), z_{0} z_{1}\right) .
\end{aligned}
$$

Proof. This follows from equations (48), (49), and Proposition 6.1

It follows from Theorem 5.5 that the Brauer-Picard groups $\operatorname{BrPic}(\mathcal{C}(D, 1))$ and $\operatorname{BrPic}(\mathcal{C}(Q, 1))$ are extensions of $\operatorname{Stab}\left(\omega_{D}\right)$ and $\operatorname{Stab}\left(\omega_{Q}\right)$, respectively, by elementary abelian $p$-groups. One can find these stabilizers using the explicit formulas (51) and (52). Below we do it for $\omega_{D}$.

Let $V$ be a finite dimensional vector space. For any subgroup $G \subset G L(V)$ define the corresponding affine group Aff $G=V \rtimes G$.

Proposition 6.3. Suppose $n>1$. Then

$$
\operatorname{Stab}\left(\omega_{D}\right) \cong \operatorname{AffS} p_{2 n}\left(\mathbb{F}_{p}\right) \rtimes \mathbb{F}_{p}^{\times} .
$$

Here $S p_{2 n}\left(\mathbb{F}_{p}\right)$ denotes the symplectic group.

Proof. Let $\left\{e_{0}, e_{1}, \ldots, e_{2 n}\right\}$ be the standard basis of $V_{2 n+1}$ so that $\left\{x_{0}, x_{1}, \ldots, x_{2 n}\right\}$ is the dual basis of $V_{2 n+1}^{*}$. By the rank of $\sum_{i} a_{i} \wedge b_{i} \in \bigwedge^{2} V^{*}$ we mean the rank of the associated linear endomorphism of $v$ given by

$$
V \rightarrow V: v \mapsto \sum_{i}\left\langle v, a_{i}\right\rangle b_{i}-\left\langle v, b_{i}\right\rangle a_{i}
$$


For every $g \in \operatorname{Stab}\left(\omega_{D}\right)$ and every $v \in V_{2 n+1}$ the ranks of $\iota_{g(v)}\left(\omega_{D}\right)$ and $\iota_{v}\left(\omega_{D}\right)$ are equal. It follows that the span of $\left\{e_{1}, \ldots, e_{2 n}\right\}$ is stable under $\operatorname{Stab}\left(\omega_{D}\right)$. Indeed, non-zero vectors $v$ lying in this span are characterized by the property that $\iota_{v}\left(\omega_{D}\right)$ has rank 2. Let $s=\sum_{i=1}^{n} x_{2 i-1} \wedge x_{2 i}$ (it corresponds to the symplectic form). An element $g \in \operatorname{Stab}\left(\omega_{D}\right)$ must map $e_{0}$ to $\lambda e_{0}+\sum_{i=1}^{2 n} v_{i} e_{i}$ and the dual of the restriction of $g$ on the span of $\left\{e_{1}, \ldots, e_{2 n}\right\}$ must map $s$ to $\lambda^{-1} s$, where $v=\left(v_{1}, \ldots v_{2 n}\right)$ is an arbitrary vector in $\mathbb{F}_{p}^{2 n}$ and $\lambda \in \mathbb{F}_{p}^{\times}$.

Thus, $\operatorname{Stab}\left(\omega_{D}\right)$ is generated by matrices of the form

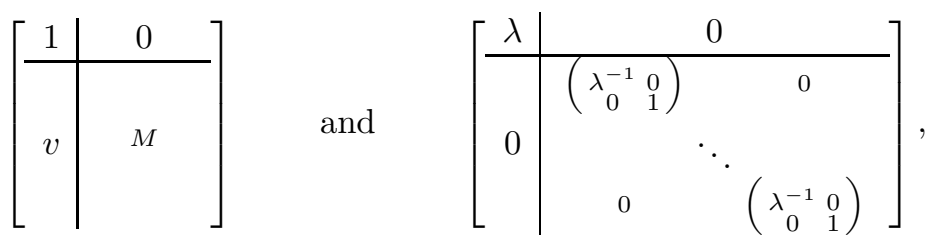

where $v \in \mathbb{F}_{p}^{2 n}, M \in S p_{2 n}\left(\mathbb{F}_{p}\right)$, and $\lambda \in \mathbb{F}_{p}^{\times}$. This clearly implies the statement.

When $n=1$, i.e., when $D, Q$ are extra special groups of order $p^{3}$, there is a neat precise description of their Brauer-Picard groups.

Proposition 6.4. Let $n=1$. Then

$$
\operatorname{Stab}\left(\omega_{D}\right)=S L_{3}\left(\mathbb{F}_{p}\right) \quad \text { and } \quad \operatorname{Stab}\left(\omega_{Q}\right)=\operatorname{AffO}\left(\mathbb{F}_{p}\right) .
$$

Proof. For any $A \in G L_{3}\left(\mathbb{F}_{p}\right)$ we have

$$
A\left(x_{0} \wedge x_{1} \wedge x_{2}\right)=\operatorname{det}(A)\left(x_{0} \wedge x_{1} \wedge x_{2}\right)
$$

in $\bigwedge^{3}\left(x_{0}, x_{1}, x_{2}\right)$, which proves the first equality. The group of matrices in $S L_{3}\left(\mathbb{F}_{p}\right)$ stabilizing $z_{0} z_{1} \in \mathrm{S}^{2}\left(z_{0}, z_{1}, z_{2}\right)$ is precisely the 2-dimensional affine orthogonal group, which proves the second equality.

Corollary 6.5. Let $D, Q$ be the extra special groups of order $p^{3}$ of exponents $p$ and $p^{2}$, respectively. Then

$$
\operatorname{BrPic}(\mathcal{C}(D, 1)) \cong S L_{3}\left(\mathbb{F}_{p}\right) \quad \text { and } \quad \operatorname{BrPic}(\mathcal{C}(Q, 1)) \cong \operatorname{AffO} O_{2}\left(\mathbb{F}_{p}\right)
$$

Proof. Note that the homomorphisms $\iota\left(\omega_{\text {alt }}\right)$ for $\omega=\omega_{D}, \omega_{Q}$ in (41) are isomorphisms, so $\operatorname{BrPic}\left(\mathcal{C}\left(V_{3}, \omega\right)\right)=\operatorname{Stab}(\omega)$ and the isomorphisms follow from Proposition 6.4.

Remark 6.6. The first of isomorphisms in (53) was established by Riepel in $\mathrm{R}$ by different methods.

6.2. Brauer-Picard groups of representation categories of extra special 2 -groups. Let $D$ and $Q$ denote extra special groups of order $2^{2 n+1}$. They can be constructed as follows. The group $D$ is the central product of $n$ copies of the dihedral group of order 8 and $Q$ is the central product $n-1$ copies of the dihedral group of order 8 and one copy of the quaternion group. 
It is straightforward to compute cohomology classes $\kappa_{D}, \kappa_{Q} \in H^{2}\left(V_{2 n}, \mathbb{F}_{2}\right)$ corresponding to extension (46) with $G=D, Q$. We have

$$
\begin{aligned}
& \kappa_{D}=\sum_{i=1}^{n} x_{2 i-1} x_{2 i}, \\
& \kappa_{Q}=\sum_{i=1}^{n} x_{2 i-1} x_{2 i}+x_{1}^{2}+x_{2}^{2},
\end{aligned}
$$

where generators $x_{i}$ are defined by (42).

Recall from Proposition 5.6 that there is an isomorphism of $G L_{2 n+1}\left(\mathbb{F}_{2}\right)$-modules:

$$
H^{3}\left(V_{2 n+1}, k^{\times}\right) \cong \mathrm{S}^{3}\left(x_{0}, x_{1}, \ldots, x_{2 n}\right) / \mathbb{F}_{2}\left\langle x_{i}^{2} x_{j}+x_{i} x_{j}^{2} \mid i, j=0, \ldots, 2 n\right\rangle .
$$

Below we identify $\omega$ with its image under (56).

Proposition 6.7. We have

$$
\begin{aligned}
& \omega_{D}=\sum_{i=1}^{n} x_{0} x_{2 i-1} x_{2 i} \\
& \omega_{Q}=\sum_{i=1}^{n} x_{0} x_{2 i-1} x_{2 i}+x_{0} x_{1}^{2}+x_{0} x_{2}^{2} .
\end{aligned}
$$

Proof. This follows from equations (54), (55), and Proposition 6.1]

Recall from Proposition 5.7 that there is a short exact sequence of $G L_{2 n+1}\left(\mathbb{F}_{2}\right)$ modules

$$
0 \rightarrow \mathrm{S}^{2}\left(x_{0}, x_{1}, \ldots, x_{2 n}\right) \rightarrow H^{3}\left(V_{2 n+1}, k^{\times}\right) \stackrel{\pi}{\rightarrow} \bigwedge^{3}\left(x_{0}, x_{1}, \ldots, x_{2 n}\right) \rightarrow 0 .
$$

Proposition 6.8. We have $\pi\left(\omega_{D}\right)=\pi\left(\omega_{Q}\right)=\omega_{\text {alt }}$, where

$$
\omega_{\text {alt }}=x_{0} \wedge\left(\sum_{i=1}^{n} x_{2 i-1} \wedge x_{2 i},\right) .
$$

Proof. The homomorphism $\pi: H^{3}\left(V_{2 n+1}, k^{\times}\right) \rightarrow \bigwedge^{3}\left(V_{2 n+1}^{*}\right)$ is described explicitly in the proof of Proposition 5.7. The result is immediate from there.

In view of Corollary 5.10 the Brauer-Picard groups of $\mathcal{C}(D, 1)$ and $\mathcal{C}(Q, 1)$ are extensions of $\operatorname{Stab}\left(\omega_{D}\right)$ and $\operatorname{Stab}\left(\omega_{Q}\right)$, respectively, by elementary abelian 2-groups. The above stabilizers can be found using the explicit formulas (57) and (58).

We consider $\operatorname{Stab}\left(\omega_{D}\right)$ and $\operatorname{Stab}\left(\omega_{Q}\right)$ as subgroups of $\operatorname{Stab}\left(\omega_{\text {alt }}\right)$.

Proposition 6.9. Suppose $n>1$. Then

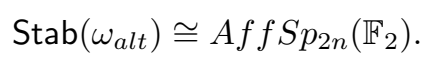

Proof. This is similar to the proof of Proposition 6.3.

Proposition 6.10. Let $n>1$. The projection from AffSpp $p_{2 n}\left(\mathbb{F}_{2}\right)$ to $\operatorname{Sp}_{2 n}\left(\mathbb{F}_{2}\right)$ induces inclusions

$$
\operatorname{Stab}\left(\omega_{D}\right) \hookrightarrow S p_{2 n}\left(\mathbb{F}_{2}\right) \quad \text { and } \quad \operatorname{Stab}\left(\omega_{Q}\right) \hookrightarrow S p_{2 n}\left(\mathbb{F}_{2}\right)
$$

Proof. A simple computation verifies that the kernel of this projection intersects both $\operatorname{Stab}\left(\omega_{D}\right)$ and $\operatorname{Stab}\left(\omega_{Q}\right)$ trivially. 
Proposition 6.11. Let $n>1$. Then

$$
\operatorname{Stab}\left(\omega_{D}\right) \cong S p_{2 n}\left(\mathbb{F}_{2}\right) \quad \text { and } \quad \operatorname{Stab}\left(\omega_{Q}\right) \cong S p_{2 n}\left(\mathbb{F}_{2}\right)
$$

Proof. Second cohomology classes $\kappa_{D}$ and $\kappa_{Q}$ defined in equations (54) and (55), represent the two equivalence classes of quadratic forms in even dimension $\mathrm{W}$, 3.4.7]. Their respective stabilizers, the orthogonal groups $O_{2 n}^{+}\left(\mathbb{F}_{2}\right)$ and $O_{2 n}^{-}\left(\mathbb{F}_{2}\right)$, are identified with subgroups of $\operatorname{Stab}\left(\omega_{D}\right)$ and $\operatorname{Stab}\left(\omega_{Q}\right)$.

It is known that $O_{2 n}^{+}\left(\mathbb{F}_{2}\right)$ and $O_{2 n}^{-}\left(\mathbb{F}_{2}\right)$ are maximal subgroups of $S p_{2 n}\left(\mathbb{F}_{2}\right)[\mathrm{P}$, Theorem 1.5].

Let $M \in G L\left(V_{2 n+1}\right)$ be defined by

$M\left(v_{0}, v_{1}, v_{2}, v_{3}, v_{4}, \ldots, v_{2 n-1}, v_{2 n}\right)=\left(v_{0}, v_{1}, v_{2}, v_{0}+v_{3}+v_{4}, v_{4}, \ldots, v_{2 n-1}, v_{2 n}\right)$.

Note that $M \in \operatorname{Stab}\left(\omega_{D}\right)$ and $M \in \operatorname{Stab}\left(\omega_{Q}\right)$, however, it is not an element of either orthogonal subgroup. Thus the images of inclusions specified in Proposition 6.10 properly contain maximal subgroups. It follows that these are isomorphisms.

Proposition 6.12. Let $n=1$. Then

$$
\operatorname{Stab}\left(\omega_{D}\right) \cong S_{4} \quad \text { and } \quad \operatorname{Stab}\left(\omega_{Q}\right)=S_{3}
$$

Proof. For any $\omega \in H^{3}\left(V_{3}, k^{\times}\right)=\mathrm{S}^{3}\left(x_{0}, x_{1}, x_{2}\right) / \mathbb{F}_{2}\left\langle x_{i}^{2} x_{j}+x_{i} x_{j}^{2} \mid i, j=0,1,2\right\rangle$ the evaluation map

$$
V_{3} \rightarrow \mathbb{F}_{2}:\left(v_{0}, v_{1}, v_{2}\right) \mapsto \omega\left(v_{0}, v_{1}, v_{2}\right)
$$

is well defined and, furthermore, $\omega$ is completely determined by the set of vectors of $V_{3}$ mapped to 1 (cf. [M]).

For $\omega=\omega_{D}=x_{0} x_{1} x_{2}$ this set consists of the single vector $(1,1,1)$. So $\operatorname{Stab}\left(\omega_{D}\right)$ is precisely the subgroup of automorphisms of $V_{3}$ fixing this vector, i.e., $\operatorname{Stab}\left(\omega_{D}\right) \cong$ Aff $G L_{2}\left(\mathbb{F}_{2}\right) \cong \mathbb{F}_{2}^{2} \rtimes G L_{2}\left(\mathbb{F}_{2}\right) \cong S_{4}$.

For $\omega=\omega_{Q}=x_{0} x_{1} x_{2}+x_{0} x_{1}^{2}+x_{0} x_{2}^{2}$ this set consists of vectors $(1,1,1),(1,1,0)$, and $(1,0,1)$. The group $\operatorname{Stab}\left(\omega_{Q}\right)$ consists of automorphisms of $V_{3}$ permuting these vectors. Since they form a basis of $V_{3}$, we conclude $\operatorname{Stab}\left(\omega_{Q}\right) \cong S_{3}$.

Corollary 6.13. Let $D, Q$ be the dihedral group and quaternion groups of order 8 , respectively. Then

$$
\operatorname{BrPic}(\mathcal{C}(D, 1)) \cong S_{4} \quad \text { and } \quad \operatorname{BrPic}(\mathcal{C}(Q, 1)) \cong S_{3} .
$$

Proof. Note that the homomorphisms $\iota\left(\omega_{\text {alt }}\right)$ for $\omega=\omega_{D}, \omega_{Q}$ in (41) are isomorphisms, so $\operatorname{BrPic}\left(\mathcal{C}\left(V_{3}, \omega\right)\right)=\operatorname{Stab}(\omega)$ and the isomorphisms follow from Proposition 6.12

Remark 6.14. The isomorphisms in (61) were established in [NR by different methods.

6.3. Pointed $p$-categories coming from metric modular Lie algebras. In view of isomorphism (34) one can produce interesting examples of 3-cocycles on elementary abelian groups as follows.

Let $F$ be a finite field of characteristic $p>3$. Below we consider finite dimensional Lie algebras over $F$. We refer the reader to $[\underline{S}$ for the theory of modular Lie algebras. 
Definition 6.15. A pre-metric Lie algebra is a Lie algebra $\mathfrak{g}$ equipped with an invariant symmetric bilinear form (, ), i.e., such that

$$
([a, b], c)=(a,[b, c])
$$

for all $a, b, c \in \mathfrak{g}$. A metric Lie algebra is a pre-metric Lie algebra such that $($,$) is$ non-degenerate.

For a Lie algebra $\mathfrak{g}$ let $\operatorname{Aut}(\mathfrak{g})$ denote the group of Lie algebra automorphisms of $\mathfrak{g}$. For a pre-metric Lie algebra $\mathfrak{g}$ let $\operatorname{Aut}_{m}(\mathfrak{g}) \subset \operatorname{Aut}(\mathfrak{g})$ denote the group of Lie algebra automorphisms of $\mathfrak{g}$ preserving $($,$) .$

Consider the following bilinear symmetric and trilinear alternating forms on $\mathfrak{g}$ :

$$
\tilde{\omega}_{\text {sym }}(a, b)=(a, b) \quad \text { and } \quad \tilde{\omega}_{a l t}(a, b, c)=([a, b], c), \quad a, b, c \in \mathfrak{g},
$$

and identify them with $\omega_{\text {sym }} \in \mathrm{S}^{2}\left(\mathfrak{g}^{*}\right)$ and $\omega_{\text {alt }} \in \bigwedge^{3}\left(\mathfrak{g}^{*}\right)$ by means of symmetrization and anti-symmetrization maps.

Let $V$ denote the underlying additive group of $\mathfrak{g}$. It is an elementary abelian $p$-group.

Set

$$
\omega=\omega_{\text {alt }}+\omega_{\text {sym }} \in H^{3}\left(V, k^{\times}\right) .
$$

Proposition 6.16. Let $\mathfrak{g}$ be a metric Lie algebra such that $\mathfrak{g}=[\mathfrak{g}$, $\mathfrak{g}]$ and let $\omega \in H^{3}\left(V, k^{\times}\right)$be the 3 -cocycle constructed above. Then $\operatorname{Stab}(\omega) \cong \operatorname{Aut}_{m}(\mathfrak{g})$.

Proof. It is clear that each $\phi \in \operatorname{Aut}_{m}(\mathfrak{g})$ stabilizes $\tilde{\omega}_{\text {sym }}$ and $\tilde{\omega}_{\text {alt }}$. Hence, it stabilizes $\omega$. Conversely, if $\psi$ is a group automorphism of $V$ stabilizing $\omega$ then it must stabilize both $\omega_{\text {sym }}$ and $\omega_{\text {alt }}$. The former condition means that $\psi$ preserves $($,$) and the latter$ one means that it is a Lie algebra homomorphism.

Proposition 6.17. Let $\mathfrak{g}$ be a pre-metric Lie algebra. Then $\omega_{\text {alt }} \in \bigwedge^{3}\left(\mathfrak{g}^{*}\right)$ is nondegenerate if and only if $\mathfrak{g}=[\mathfrak{g}, \mathfrak{g}]$ and $($,$) is non-degenerate (i.e., \mathfrak{g}$ is a metric Lie algebra).

Proof. Non-degeneracy of $\omega_{\text {alt }}$ is equivalent to non-degeneracy of the alternating trilinear form $\tilde{\omega}_{\text {alt }}$. This implies the statement.

Corollary 6.18. Suppose that $\mathfrak{g}$ is a metric Lie algebra such that $\mathfrak{g}=[\mathfrak{g}, \mathfrak{g}]$. There is an exact sequence

$$
0 \rightarrow V \rightarrow \bigwedge^{2}\left(V^{*}\right) \rightarrow \operatorname{BrPic}(\mathcal{C}(V, \omega)) \rightarrow \operatorname{Aut}_{m}(\mathfrak{g}) \rightarrow 0 .
$$

Proof. This follows from Theorem 5.5 and Propositions 6.16 and 6.17

One can take $(,$,$) to be the Killing form of \mathfrak{g}$ :

$$
(a, b)=\operatorname{Tr}_{\mathfrak{g}}(\operatorname{ad}(a) \operatorname{ad}(b)), \quad a, b \in \mathfrak{g},
$$

where ad denotes the adjoint representation of $\mathfrak{g}$. Let $\omega^{\mathfrak{g}} \in H^{3}\left(V, k^{\times}\right)$denote the corresponding 3rd cohomology class defined by (62).

Remark 6.19. If $\mathfrak{g}$ has a non-degenerate Killing form then $\mathfrak{g}$ is a direct sum of simple Lie algebras. Unlike for Lie algebras over $\mathbb{C}$, the converse to this statement is false in positive characteristic. All simple classical Lie algebras with a nondegenerate Killing over a field $F$ with $\operatorname{char}(F)>3$ are known. The necessary and sufficient condition is that $\operatorname{char}(F)$ should not divide the determinant of the Killing form of the corresponding simple complex Lie algebra, see [ $\underline{\mathrm{S}}$, Chapter II $\S 9]$. 
It can even happen that every trace form of a simple $\mathfrak{g}$ is degenerate, this is the case, e.g., for $\mathfrak{g}=\mathfrak{s l}_{n}(F)$ when $\operatorname{char}(F)$ divides $n$.

Proposition 6.20. Let $\mathfrak{g}$ be a simple Lie algebra with a non-degenerate Killing form. We have an exact sequence

$$
0 \rightarrow V \rightarrow \bigwedge^{2}\left(V^{*}\right) \rightarrow \operatorname{BrPic}\left(\mathcal{C}\left(V, \omega^{\mathfrak{g}}\right)\right) \rightarrow \operatorname{Aut}(\mathfrak{g}) \rightarrow 0
$$

Proof. It is clear that when (, ) given by the Killing form of $\mathfrak{g}$ we have $\operatorname{Aut}_{m}(\mathfrak{g})=$ Aut $(\mathfrak{g})$. The result follows from Corollary 6.18.

Remark 6.21. The automorphism groups of classical simple modular Lie algebras $\mathfrak{g}$ are known [S].

Thus, finite simple groups of Lie type naturally appear as composition factors of groups of autoequivalences and Brauer-Picard groups of pointed fusion categories.

Example 6.22. Take $\mathfrak{g}=\mathfrak{s l}_{2}(F)$. Since $p$ is odd, the Killing form is non-degenerate. Let

$$
e=\left[\begin{array}{ll}
0 & 1 \\
0 & 0
\end{array}\right], \quad f=\left[\begin{array}{ll}
0 & 0 \\
1 & 0
\end{array}\right], \quad \text { and } \quad h=\left[\begin{array}{cc}
1 & 0 \\
0 & -1
\end{array}\right]
$$

be a basis of $\mathfrak{g}$ so that

$\operatorname{ad}(e)=\left[\begin{array}{ccc}0 & 0 & -2 \\ 0 & 0 & 0 \\ 0 & 1 & 0\end{array}\right], \operatorname{ad}(f)=\left[\begin{array}{ccc}0 & 0 & 0 \\ 0 & 0 & 2 \\ -1 & 0 & 0\end{array}\right]$, and $\operatorname{ad}(h)=\left[\begin{array}{ccc}2 & 0 & 0 \\ 0 & -2 & 0 \\ 0 & 0 & 0\end{array}\right]$.

The corresponding 3-cocycle $\omega^{\mathfrak{g}}$ is given by

$$
\omega^{\mathfrak{g}}=\left(8 x_{e} \wedge x_{f} \wedge x_{h}, 4 z_{e} z_{f}+8 z_{h}^{2}\right) .
$$

In particular, when $F=\mathbb{F}_{p}$ we have $\operatorname{BrPic}\left(\mathcal{C}\left(V, \omega^{\mathfrak{g}}\right)\right)=S O_{3}\left(\mathbb{F}_{p}\right)$.

6.4. Representation category of the Kac-Paljutkin Hopf algebra. Recall that the Kac-Paljutkin Hopf algebra KP is the unique non-commutative and noncocommutative semisimple Hopf algebra of dimension $8[\mathrm{KP}$.

Let $\mathcal{K P}$ be the fusion category of representations of $K P$. Below we compute the Brauer-Picard group of $\mathcal{K} \mathcal{P}$.

Note that $\mathcal{K \mathcal { P }}$ is a particular case of a Tambara-Yamagami fusion category [TY]. Recall that such categories $\mathcal{T} \mathcal{Y}(A, \chi, \tau)$ are defined as follows. Let $A$ be a finite abelian group. The set of simple objects of $\mathcal{T} \mathcal{Y}(A, \chi, \tau)$ is $\{a \mid a \in A\} \cup\{m\}$ with tensor products given by

$$
a \otimes b=a b, \quad a \otimes m=m \otimes a=m, \quad m \otimes m=\bigoplus_{a \in A},
$$

and associativity constraints determined by a non-degenerate symmetric bilinear form $\chi: A \otimes A \rightarrow k^{\times}$and $\tau \in k$ such that $\tau^{2}=|A|^{-1}$ (see TY] for details of the definition). It was shown in [TY] Section 4] that

$$
\mathcal{K} \mathcal{P} \cong \mathcal{T} \mathcal{Y}\left(\mathbb{Z}_{2} \times \mathbb{Z}_{2}, \chi, \frac{1}{2}\right)
$$

where $\chi$ is the bilinear form on $\mathbb{Z}_{2} \times \mathbb{Z}_{2}=\{1, a, b, c\}$ determined by

$$
\chi(a, a)=1, \quad \chi(b, b)=\chi(c, c)=-1, \quad \chi(b, c)=1 .
$$

The centers of Tambara-Yamagami categories and their $S$ - and $T$-matrices were explicitly described in [GNN, Section 4C]. The following result is immediate from that description. 
Lemma 6.23. The forgetful homomorphism

$$
\operatorname{Inv}(\mathcal{Z}(\mathcal{T Y}(A, \chi, \tau))) \rightarrow \operatorname{Inv}(\mathcal{T Y}(A, \chi, \tau))
$$

is surjective.

Let $D$ and $Q$ denote, respectively, the dihedral and quaternion groups of order 8 . We will use the following presentation of $D$ by generators and relations:

$$
D=\left\langle r, s \mid r^{4}=s^{2}=1, r s=s r^{-1}\right\rangle .
$$

Lemma 6.24. (i) $\mathcal{Z}(\mathcal{K} \mathcal{P})_{p t} \cong \operatorname{Rep}\left(\mathbb{Z}_{2}^{3}\right)$ is a symmetric non-Tannakian category.

(ii) $\mathcal{Z}(\mathcal{K P})$ has precisely two Lagrangian subcategories; both of them are equivalent to $\operatorname{Rep}(D)$.

(iii) $\mathcal{K P}$ has a unique, up to equivalence, fiber functor (i.e., a tensor functor to $\mathrm{Vec})$.

Proof. Using the description of $\mathcal{Z}(\mathcal{K P})$ from [GNN] and equivalence [64) one sees that every invertible object $x$ of $\mathcal{K P}$ has precisely 2 structures of an object of $\mathcal{Z}(\mathcal{K P})$ and the square of the braiding between objects supported on invertible objects $x, y \in A$ is $\chi(x, y) \operatorname{id}_{x y}$. This proves (i).

Inspecting the $S$ - and $T$-matrices of $\mathcal{Z}(\mathcal{T Y}(A, \chi, \tau))$ we conclude that Lagrangian subcategories of $\mathcal{Z}(\mathcal{K P})$ are precisely those that contain 4 invertible objects supported on $1, a$ and one two-dimensional simple object supported either on $1 \oplus a$ or on $b \oplus c$. So there are two such subcategories. Since the dihedral group $D$ and the quaternionic group $Q$ are the only non-abelian groups of order 8 the above Lagrangian categories must be equivalent to either $\operatorname{Rep}(D)$ or $\operatorname{Rep}(Q)$. The latter case leads to a contradiction: it implies that $\mathcal{Z}(\mathcal{K} \mathcal{P})=\mathcal{Z}(\mathcal{C}(Q, \eta))$ for some nontrivial $\eta \in H^{3}\left(Q, k^{\times}\right)$. But $\mathcal{C}(Q, \eta)$ is not categorically Morita equivalent to the representation category of a Hopf algebra by [O. This proves (ii).

Finally, (iii) follows for the classification of fiber functors on Tambara-Yamagami categories $[\mathrm{T}]$.

Lemma 6.25. Let $\mathcal{C}$ be a fusion category categorically Morita equivalent to $\mathcal{K} \mathcal{P}$. Then either $\mathcal{C} \cong \mathcal{K P}$ or $\mathcal{C} \cong \mathcal{C}(D, \omega)$ for some non-trivial 3-cocycle $\omega \in H^{3}\left(D, k^{\times}\right)$.

Proof. If $\mathcal{C}$ is not pointed then it must be a Tambara-Yamagami category. Combining Lemmas 6.23 and 6.24(i) we conclude that $\operatorname{lnv}(\mathcal{C})=\mathbb{Z}_{2} \times \mathbb{Z}_{2}$. It is proved in [NN Section 5.2] that the only fusion category with the group of invertible objects $\mathbb{Z}_{2} \times \mathbb{Z}_{2}$ categorically Morita equivalent to $\mathcal{K} \mathcal{P}$ is $\mathcal{K} \mathcal{P}$ itself.

If $\mathcal{C}$ is pointed then it corresponds to a Lagrangian subcategory in $\mathcal{Z}(\mathcal{K P})$ and the statement follows from Lemma 6.24(ii).

Using Lemma 6.24(iii), description of $\omega$ from $\underline{\mathrm{KP}}$, and classification of fiber functors on group-theoretical categories $[\mathrm{O}$ ] one can list all indecomposable $\mathcal{C}(D, \omega)$ module categories (and, hence, all indecomposable $\mathcal{K} \mathcal{P}$-module categories). There are precisely 6 such categories, parameterized by conjugacy classes of subgroups of $D$ on which $\omega$ has trivial restriction, namely: $1,\left\langle r^{2}\right\rangle,\left\langle r^{2}, s\right\rangle,\langle s r\rangle,\left\langle r^{2}, s r\right\rangle,\langle s\rangle$.

Remark 6.26. The same list of $\mathcal{K} \mathcal{P}$-module categories can be obtained using the classification of module categories over Tambara-Yamagami categories from [MM]. We are grateful to Ehud Meir for sharing his calculations with us. 
Corollary 6.27. There are precisely 2 equivalence classes of indecomposable $\mathcal{K} \mathcal{P}$ module categories such that the dual fusion category is pointed and precisely 4 equivalence classes of indecomposable $\mathcal{K} \mathcal{P}$-module categories such that the dual fusion category is equivalent to $\mathcal{K} \mathcal{P}$.

Proof. Module $\mathcal{K} \mathcal{P}$-categories with pointed duals correspond to Lagrangian subcategories of $\mathcal{Z}(\mathcal{K P})$. Since there are 6 classes of indecomposable $\mathcal{K} \mathcal{P}$-module categories, the result follows from Lemma 6.24 (ii)

Corollary 6.28. $|\operatorname{BrPic}(\mathcal{K P})|=8$.

Proof. By Corollary $6.27 \mathcal{K P}$ has 4 module categories with the dual $\mathcal{K} \mathcal{P}$, so the statement follows from Proposition 3.6 and the fact that $\operatorname{Aut}(\mathcal{K P})=\mathbb{Z}_{2}[\mathrm{~T}]$.

Corollary 6.29. The image of the induction homomorphism

$$
\operatorname{Aut}(\mathcal{C}(D, \omega)) \rightarrow \operatorname{Aut}^{b r}(\mathcal{Z}(\mathcal{C}(D, \omega)))=\operatorname{Aut}^{b r}(\mathcal{Z}(\mathcal{K} \mathcal{P}))
$$

is isomorphic to $\mathbb{Z}_{2} \times \mathbb{Z}_{2}$.

Proof. By Remark 3.7 (see also $[\mathrm{NR}) \operatorname{Aut}^{\text {br }}(\mathcal{Z}(\mathcal{K P}))$ acts transitively on Lagrangian subcategories of $\mathcal{Z}(\mathcal{K P})$ and the image of $(65)$ is precisely the stabilizer of a Lagrangian subcategory $\operatorname{Rep}(D)$. Hence, this image must contain 4 elements by Corollary 6.28. These elements are the autoequivalences of the form ind $\left(F_{a, \mu}\right)$, where $a \in \operatorname{Stab}(\omega)$ and $\mu$ is a 2-coboundary such that

$$
d^{2} \mu=\frac{\omega \circ(a \times a \times a)}{\omega},
$$

see Section 2.3. Note that $\operatorname{Out}(D) \cong \mathbb{Z}_{2}$, where the class of non-trivial outer automorphism is represented by $a \in \operatorname{Aut}(D)$ such that $a(r)=r, a(s)=s r$.

Consider the action of the above autoequivalences ind $\left(F_{a, \mu}\right)$ on $\mathcal{Z}(\mathcal{C}(D, \omega))_{p t}$. The invertible objects of the center are supported on $\left\{1, r^{2}\right\}$. One easily checks that autoequivalences $\operatorname{ind}\left(F_{1, \mu}\right)$ fix central objects supported on 1 and non-trivially permute those supported on $r^{2}$, while autoequivalences ind $\left(F_{a, \mu}\right)$ non-trivially permute central objects supported on 1 and have order 2. Thus, the induced autoequivalences form a group isomorphic to $\mathbb{Z}_{2} \times \mathbb{Z}_{2}$.

Proposition 6.30. $\operatorname{BrPic}(\mathcal{K P}) \cong \mathbb{Z}_{2} \times \mathbb{Z}_{2} \times \mathbb{Z}_{2}$.

Proof. Consider the Picard induction homomorphism (see Section 2.5)

$$
\operatorname{Pic}\left(\mathcal{Z}(\mathcal{K} \mathcal{P})_{p t}\right) \rightarrow \operatorname{Pic}(\mathcal{Z}(\mathcal{K} \mathcal{P}))=\operatorname{BrPic}(\mathcal{K P}): \mathcal{M} \mapsto \mathcal{Z}(\mathcal{K P}) \bigotimes_{\mathcal{Z}(\mathcal{K} \mathcal{P})_{p t}} \mathcal{M}
$$

By Lemma 6.24(i) and the result of $\left[\mathrm{C}\right.$ ] we have $\operatorname{Pic}\left(\mathcal{Z}(\mathcal{K P})_{p t}\right)=\mathbb{Z}_{2}^{4}$. On the other hand, by (11) the kernel of (66) coincides with the universal grading group of $\mathcal{Z}(\mathcal{K P})$, i.e., is isomorphic to $\mathbb{Z}_{2}^{3}$. Thus, the image of (66) has order 2. Let $\alpha \in \operatorname{Aut}^{b r}(\mathcal{Z}(\mathcal{K P}))$ correspond to the non-trivial element of this image. Since $\alpha$ induces the trivial autoequivalence of $\mathcal{Z}(\mathcal{K P})_{p t}$, it is not in the image of (65), cf. the proof of Corollary 6.29. Hence, $\alpha$ must permute the two Lagrangian subcategories of $\mathcal{Z}(\mathcal{K} \mathcal{P})$. Since these subcategories are fixed by the image of (65) we get an injective homomorphism from $\operatorname{Aut}^{b r}(\mathcal{K} \mathcal{P})$ to $\mathbb{Z}_{2}^{3}$ and the result follows from Corollary 6.28 


\section{REFERENCES}

[A] A. Adem, Cohomology of finite groups, 2nd edition, Springer, (2004).

[BN] C. Bontea, D. Nikshych, On the Brauer-Picard group of a finite symmetric tensor category, J. Algebra, 440 (2015), 187-218.

[C] G. Carnovale The Brauer group of modified supergroup algebras, J. Algebra 305 (2006), no. 2, 993-1036.

[DPR] R. Dijkgraaf, V. Pasquier, and P. Roche, Quasi-Hopf algebras, group cohomology, and orbifold models, Nuclear Phys. B, Proc. Suppl., 18, (1991), no. 2, 60-72.

[DGNO1] V. Drinfeld, S. Gelaki, D. Nikshych, and V. Ostrik, Group-theoretical properties of nilpotent modular categories. e-print arXiv:0704.0195 (2007).

[DGNO2] V. Drinfeld, S. Gelaki, D. Nikshych, and V. Ostrik, On braided fusion categories I, Selecta Mathematica, 16 (2010), no. 1, 1-119.

[DMNO] A. Davydov, M. Müger, D. Nikshych, and V. Ostrik, The Witt group of non-degenerate braided fusion categories, Journal für die reine und angewandte Mathematik, 677 (2013), $135-177$.

[EGNO] P. Etingof, S. Gelaki, D. Nikshych, V. Ostrik, Tensor categories, Mathematical Surveys and Monographs, 205, American Mathematical Society (2015).

[ENO1] P. Etingof, D. Nikshych, and V. Ostrik, Fusion categories and homotopy theory, Quantum Topology, 1 (2010), no. 3, 209-273.

[ENO2] P. Etingof, D. Nikshych, and V. Ostrik, Weakly group-theoretical and solvable fusion categories, Adv. Math, 226 (2011), no. 1, 176-205

[GP] C. Galindo, J. Plavnik, Tensor functor between Morita duals of fusion categories, e-print arXiv:1407.2783 (2014).

[GNN] S. Gelaki, D. Naidu, and D. Nikshych, Centers of graded fusion categories, Algebra and Number Theory, 3 (2009), no. 8, 959-990.

[GN] S. Gelaki, D. Nikshych, Nilpotent fusion categories. Advances in Mathematics, 217 (2008), no. 3, 1053-1071.

[GMN] C. Goff, G. Mason, and S.-H. Ng, On the gauge equivalence of twisted quantum doubles of elementary abelian and extra-special 2-groups. J. Algebra, 312 (2007), no. 2, 849-875.

[KP] G. Kac, V. Paljutkin. Finite ring groups, Trans. Moscow Math. Soc., (1966), 251-294.

[LP] S. Lentner, J. Priel, A decomposition of the Brauer-Picard group of the representation category of a finite group, e-print arXiv:1506.07832 (2015).

[M] G. Mason, Reed-Muller codes, the fourth cohomology group of a finite group, and the $\beta$ invariant, J. Algebra, 312 (2007), no. 1, 218-227.

[MM] E. Meir, E. Musicantov, Module categories over graded fusion categories, J. Pure and Applied Algebra, 216 (2012), no. 11, 2449-2466.

[MN1] G. Mason, S.-H. Ng, Group Cohomology and Gauge Equivalence of some Twisted Quantum Doubles, Trans. Amer. Math. Soc., 353 (2001), 3465-3509.

[MN2] G. Mason, S.-H. Ng, Cleft extensions and quotients of twisted quantum doubles, in Developments and retrospectives in Lie theory, Developments in Mathematics 38 (2014), 229-246.

[N] D. Naidu, Categorical Morita equivalence for group-theoretical categories, Comm. Algebra 35, (2007), no. 11, 3544-3565.

[NN] D. Naidu, D. Nikshych, Lagrangian subcategories and braided tensor equivalences of twisted quantum doubles of finite groups, Comm. Math. Phys., 279 (2008), 845-872.

[NNW] D. Naidu, D. Nikshych, and S. Witherspoon, Fusion subcategories of representation categories of twisted quantum doubles of finite groups, International Mathematics Research Notices (2009), no. 22, 4183-4219.

[NR] D. Nikshych, B. Riepel, Categorical Lagrangian Grassmannians and Brauer-Picard groups of pointed fusion categories, J. Algebra, 411 (2014), 191-214.

[O] V. Ostrik, Module categories over the Drinfeld double of a finite group. Int. Math. Res. Not. (2003), 1507-1520.

[P] H. Pollatsek, First cohomology groups of some linear groups over fields of characteristic two, Illinois J. Math., 15 (1971), no. 3, 393-417

[R] B. Riepel, Brauer-Picard groups of pointed fusion categories, Ph.D. Thesis, University of New Hampshire (2014).

[S] G.B. Seligman, Modular Lie Algebras, Springer, (1967). 
[T] D. Tambara, Representations of tensor categories with fusion rules of self-duality for abelian groups, Israel J. Math., 118 (2000), no. 1, 29-60.

[TY] D. Tambara, S. Yamagami, Tensor categories with fusion rules of self-duality for finite abelian groups, J. Algebra, 209 (1998), no. 2, 692-707.

$[\mathrm{U}]$ B. Uribe, On the classification of pointed fusion categories up to weak Morita equivalence, e-print arXiv:1511.05522 (2015).

[W] R.A. Wilson, The finite simple groups, Springer, (2009).

Department of Mathematics and Statistics, University of New Hampshire, Durham, NH 03824, USA

E-mail address: isg3@wildcats.unh.edu

Department of Mathematics and Statistics, University of New Hampshire, Durham, NH 03824, USA

E-mail address: dmitri.nikshych@unh.edu 